\title{
Many-body calculations of positron scattering and annihilation from noble-gas atoms
}

\author{
V A Dzuba $\dagger$, V V Flambaum $\dagger \ddagger$, G F Gribakin $\dagger$ and W A King $\dagger \S$ \\ $\dagger$ School of Physics, University of New South Wales, Sydney 2052, Australia \\ $\ddagger$ ITAMP, Smithsonian Observatory and Harvard University, 60 Garden Street, Cambridge, MA \\ 02138, USA
}

Received 24 March 1996, in final form 13 May 1996

\begin{abstract}
We have applied many-body theory methods to study the interaction of low-energy positrons with noble-gas atoms. The positron-atom correlation potential includes explicitly the contribution from the target polarization by the positron and that from the virtual positronium (Ps) formation. It is demonstrated that the correlations and Ps formation (or tunnelling of electrons from the atom to the positron) create virtual levels in the positron-atom system. The existence of the virtual levels strongly influences the scattering and increases the positron annihilation rate by up to 400 times. The calculated elastic cross sections are in good agreement with experimental data. The inclusion of virtual Ps formation greatly improves the agreement with experimental annihilation rates with respect to calculations taking only the polarization into account. Our calculations have highlighted the difference between the calculation of positronatom scattering and the calculation of corresponding annihilation rates. The annihilation rate is very sensitive to the behaviour of the wavefunction at small positron-electron separations. A simple approximate formula based on the Sommerfeld factor is suggested to account for the effect of electron-positron Coulomb attraction on the annihilation rates.
\end{abstract}

\section{Introduction}

The aim of this work is to apply many-body theory methods to the calculation of low-energy positron scattering from $\mathrm{Ne}, \mathrm{Ar}, \mathrm{Kr}$ and $\mathrm{Xe}$ atoms and the positron annihilation rates in lowdensity atomic gases of $\mathrm{H}, \mathrm{He}, \mathrm{Ne}, \mathrm{Ar}, \mathrm{Kr}$ and $\mathrm{Xe}$. The interaction of the positron with the atom is described by means of a non-local energy-dependent correlation potential. We show that there are two physically distinct mechanisms: polarization of the target by the projectile and virtual positronium (Ps) formation, which contribute to the correlation potential. We have calculated both of these explicitly. In all cases the Ps-formation contribution is large, and its inclusion is necessary to achieve agreement with experimental elastic cross sections. It turns out, however, that despite the good agreement of our solution with the scattering problem, an accurate calculation of the annihilation rate (or $Z_{\text {eff }}$, see section 4) requires a more elaborate approach to describe the behaviour of the wavefunction at small positronelectron separations. In other words, corrections to the electron-positron annihilation vertex due to the electron-positron Coulomb interaction should be taken into account. Beside the many-body calculations, we present a simple formula to approximate the effects of Coulomb enhancement on the annihilation rates. We suggest that this formula can be used to estimate

$\S$ Present address: Computational Molecular Biology and Drug Design Group, John Curtin School of Medical Research, Australian National University, Canberra 0200, Australia. 
the $Z_{\text {eff }}$ values of atoms and molecules, where proper calculations are not available or feasible.

The theoretical investigation of positron scattering by noble-gas atoms and the annihilation rates of positrons in noble gases is interesting for a number of reasons. Although the electronic structure of noble gases is rather simple, the phenomena that have been observed (scattering cross sections and annihilation rates) demonstrate that the description of a positron-atom system is quite complicated. It is well known that for $\mathrm{Ar}, \mathrm{Kr}$ and $\mathrm{Xe}$, the total electron scattering cross sections possess Ramsauer-Townsend minima at low electron energies. This is due to the polarization of the target by the projectile, which is a manifestation of the electron correlations. In the case of positron scattering one would expect the correlations to be even larger, due to the attraction between the positron and the electrons. Indeed, despite the static positron-atom potential being repulsive, the total scattering cross sections for positrons also possess Ramsauer-Townsend minima. Further evidence of the large correlation effects can be seen by examining the annihilation rates of positrons in noble gases. The experimentally determined annihilation rate of positrons in a dilute xenon gas is almost 300 times larger than the annihilation rate found using a static approximation (see below).

It has been shown (see, e.g., Amusia et al 1976, Basu et al 1989, 1990, Dzuba et al 1993, Higgins and Burke 1993, Mitroy 1994, Gribakin and King 1994) that to describe the positron-atom interaction accurately one needs to include the effect of virtual Ps formation. Apart from calculations of $\mathrm{He}$ (the most accurate of them are probably those done by the variational method (Humberston 1973); Amusia et al (1976) and Gribakin and King (1994) used many-body theory methods, which can hardly compete in accuracy with variational methods for simple targets like $\mathrm{H}$ and $\mathrm{He}$ ) none of the calculations of positron scattering from noble gases and of positron annihilation rates in noble gases (McEachran et al 1977, 1979, 1980, Schrader and Svetic 1982) have included the effects of virtual Ps formation, except the recent work of Dzuba et al (1993). The former calculations employed a polarizedorbital approximation which implies a linear response of the atom to the positron Coulomb field and the use of the adiabatic approximation for the positron. Because of this, the good agreement they obtained with the experimental scattering cross sections remains to some extent a puzzle. In the work of Dzuba et al (1993) the contribution of virtual Ps formation was estimated as an attraction which results from tunnelling of the electron from the atom to the positron. If the energy of the positron-atom system is close to that of the ion and Ps, such tunnelling has a resonant character and its effect can be quite substantial (Kuchiev 1992). Dzuba et al (1993) also highlighted the role of virtual levels formed by positrons with heavier noble-gas atoms.

The experimental annihilation rates for the lighter noble gases are very well established (Coleman et al 1975, Canter and Roellig 1975, Griffith and Heyland 1978). Coleman et al (1975) measured the annihilation rates for all the noble-gas atoms. Apart from Xe, the accepted values of the annihilation rates have not changed significantly over time. There have been several experimental studies on positron annihilation rates in Xe at densities down to about 1 Amagat $\dagger$ (Coleman et al 1975, Wright et al 1985, Tuomisaari et al 1988) each suggesting that the $Z_{\text {eff }}$ value of Xe is no greater than 320. In all experiments the annihilation rate showed a strong nonlinear density dependence, which hindered the determination of the single-atom annihilation rate. The reason for this behaviour, present even at densities of about 1 Amagat, is that the positron-Xe scattering length is very large and is comparable to interatomic distances, thus multiple positron-atom collisions and/or effects of clustering

$\dagger 1$ Amagat $=2.69 \times 10^{19} \mathrm{~cm}^{-3}$; the average distance between atoms at this density is about 60 au. 
can be essential (see below). Murphy and Surko (1990) measured the positron annihilation rate in Xe gas using a trap at Xe densities nine orders of magnitude less than in the previous experiments. This allowed them to avoid any many-atom effects, which could have been seen in the previous experiments. The value they obtained was $401 \pm 20$, which is $25 \%$ higher than any of the previous experiments. To date this discrepancy is unaccounted for.

There has been quite a lot of theoretical interest in the positron annihilation in noble-gas atoms (McEachran et al 1977, 1978a, b, 1979, 1980, Schrader and Svetic 1982). McEachran and co-authors obtained the $Z_{\text {eff }}$ values of 4.02, 6.99, 30.5, 56.3 and 200, for $\mathrm{He}, \mathrm{Ne}, \mathrm{Ar}$, $\mathrm{Kr}$ and $\mathrm{Xe}$ at room temperature. These numbers, Xe excepted, are in surprisingly good agreement with the experimental $Z_{\text {eff }}$ values that are presented in table 2 and with the result of an accurate variational calculations for $\mathrm{He}, Z_{\text {eff }}=3.86$ (Humberston 1974). However, apart from the latter, none of the calculations accounted for the virtual Ps formation.

Many-body theory enables us to systematically approach the problem of describing the positron-atom interaction. The perturbation series for a many-body system is very complicated. Throughout the paper we will be using a diagrammatic language to write down the various contributions to the positron-atom interaction, thus simplifying the description of the many terms of the perturbation series. The diagrammatic language also allows us to identify the various physical processes that contribute to the interaction and determine the magnitude of their relative contributions (by actually calculating the underlying mathematical expressions).

It is worth mentioning that there is an essential difference between the processes of positron-atom scattering and annihilation. In the scattering process, the positron is excluded from the inside of the atom, due to the repulsion of the nucleus. Furthermore, the polarization of the atom by the projectile and the virtual Ps formation take place at large positron-atom distances (the radius of the Ps atom is $2 \mathrm{au}$ ). Therefore, the region $r>r_{\mathrm{a}}$ (where $r$ is the position of the positron and $r_{\mathrm{a}}$ is the atomic radius) is the most important in the calculation of the scattering amplitude. However, the positron-atom annihilation rates are determined by the behaviour of the total wavefunction at very small positron-electron separations. Of course, within the many-body approach one does not deal with the total many-particle wavefunction, but rather with various amplitudes which contribute to the process under consideration. The physical distinction between the scattering and annihilation outlined above means that the role of different diagrams can be different in these two processes. Thus, we will see that the treatment of virtual Ps formation adopted in the paper is quite good for the scattering problem but insufficient for the annihilation problem. Trying to explain and resolve this problem we have found an effective way of taking into account the electron-positron Coulomb interaction in the calculation of $Z_{\text {eff }}$. Overall, by considering both the scattering and the annihilation problem we get a much deeper insight into the physics of the positron-atom interaction.

\section{Calculation of the correlation potential}

In many-body perturbation theory the projectile-atom interaction can be described by a non-local energy-dependent correlation potential (sometimes called the optical potential), which is equal to the self-energy of the single-particle Green function of the atom $\Sigma_{E}$ (Bell and Squires 1959). The quasi-particle wavefunction $\psi$ which describes the scattering of a positron off an atom then satisfies the equation

$$
\left(H_{0}+\Sigma_{E}\right) \psi=E \psi
$$


where $H_{0}$ is the zeroth-order Hamiltonian of the atom, $E$ is the energy of the projectile and $\Sigma_{E} \psi$ is given by

$$
\Sigma_{E} \psi=\int \Sigma_{E}\left(\boldsymbol{r}, \boldsymbol{r}^{\prime}\right) \psi\left(\boldsymbol{r}^{\prime}\right) \mathrm{d} \boldsymbol{r}^{\prime}
$$

Equation (1) is sometimes called the Dyson equation (see, e.g., Migdal 1967).

The usual and probably the best choice of $H_{0}$ is made by using the Hartree-Fock Hamiltonian of the atom. This Hamiltonian describes the interaction of the projectile with the static charge distribution of the ground-state atom. Its eigenstates provide a complete basis set for the subsequent calculation of correlation effects. The correlation potential $\Sigma_{E}$ is then given by a perturbation theory series in powers of the residual Coulomb interaction between the projectile and the atomic electrons and between the atomic electrons. The main contribution to $\Sigma_{E}$ is given by the lowest, second-order diagram (figure 1(a); it is convenient to show diagrams for the matrix element of the correlation potential between the initial and final projectile states $\left\langle f\left|\Sigma_{E}\right| i\right\rangle$ rather than for $\Sigma_{E}\left(\boldsymbol{r}, \boldsymbol{r}^{\prime}\right)$ itself). It was shown (see, e.g., Kelly 1963, Amusia and Cherepkov 1975) that in electron scattering, besides the second-order diagrams, several important higher-order corrections (figures $1(b)$ and $(c)$ ) should also be taken into account (these diagrams are characterized as having two interactions between the projectile and the target atom), as well as the associated exchange diagrams. The correlation potential that results from the summation of these diagrams, hereafter we will call the polarization potential, $\Sigma_{E}^{\mathrm{pol}}$. It describes the polarization of the atom by the projectile in all multipoles. For small $E$ it has the same long-range behaviour as the well known local dipole polarization potential $-\alpha / 2 r^{4}, \alpha$ being the static dipole polarizability of the atom. The potential thus calculated describes accurately electron scattering and negative ion problems (see, e.g., Amusia et al

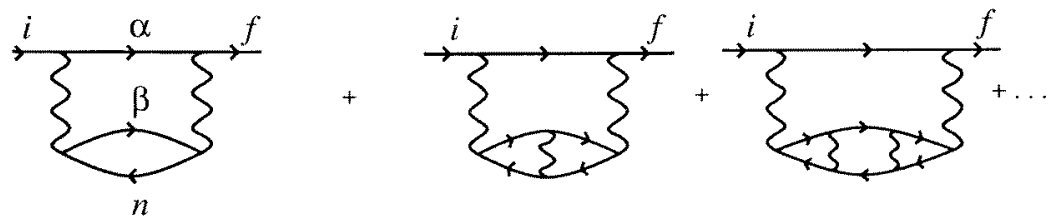

(a)

(b)

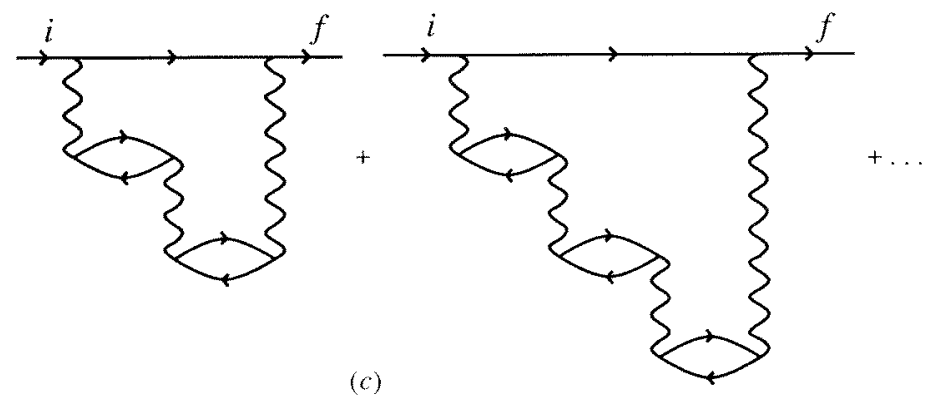

Figure 1. Direct diagrams contributing to the atomic polarization potential: (a) lowest-order diagram, $(b)$ hole-particle interaction diagrams and $(c)$ screening diagrams. As usual, the line with an arrow to the right describes a particle state (above the Fermi level, i.e. above the atomic ground state) and the line with an arrow to the left is a hole state (below the Fermi level). Summation over all intermediate states is assumed. Wavy lines are the Coulomb interactions between the projectile and target electrons or between the target electrons. The upper lines in the diagrams correspond to the projectile. 
1982, Chernysheva et al 1988, Gribakin et al 1990, Dzuba et al 1991, Dzuba and Gribakin 1994a, b).

The numerical calculation of $\Sigma_{E}^{\mathrm{pol}}$ can be performed in different ways (see the papers cited above). The series of time-forward diagrams like that in figure 1 corresponding to a given hole orbital $n$ can be taken into account by calculating the electron wavefunction in the loop of figure 1(a) in the field of this hole (Amusia and Cherepkov 1975). The summation over the intermediate states can be performed directly, by appropriately discretizing the projectile and excited electron continuum. A better numerical accuracy, however, is achieved when the Feynman diagram technique and the Green functions are used (Dzuba et al 1987, 1989). We adopt this method in the present paper. It is outlined diagrammatically in figure 2. Firstly, the polarization operator for $\omega$ energy, that includes the infinite series of hole-particle interactions (figure $2(a)$ ) is calculated. This operator is then used to calculate the polarization operator that includes the hole-particle and screening diagrams as in figure $2(b)$. This operator can then be introduced into the second-order diagram together with the Green function of $\varepsilon+\omega$ energy and integration over $\omega$ performed, as in figure 3(c). Thus, all direct diagrams shown in figure 1 and higher-order terms of the same structure are taken into account (including those with different time ordering of the electron-hole loops in the diagrams of figure $1(c)$ ). Note that chaining of the polarization operator (figure $2(b)$ ) corresponds to the RPA approximation for the dynamic response of the atom to the external electric field of the projectile.

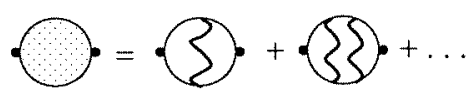

(a)

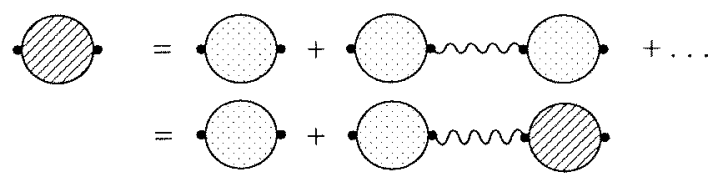

(b)

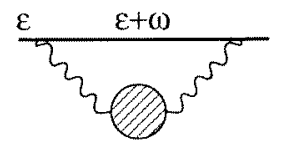

(c)

Figure 2. Diagrammatic description of the calculation of the polarization potential: $(a)$ firstly, the hole-particle interaction is taken into account in the polarization operator, $(b)$ then the screening of the Coulomb interaction; $(c)$ the second-order diagram, taking into account the hole-particle interaction and screening diagrams.

For positron-atom scattering $\Sigma_{E}^{\mathrm{pol}}$ includes only the diagrams in figure 1 and alike, as naturally there are no exchange diagrams. Of course, this does not mean that this potential is equal to the contribution of these diagrams in the electron-atom case, since the Hartree-Fock states and Green functions of the projectile which enter the expressions for the diagrams are different for positrons and electrons. Quite unexpectedly, it turned out that this approximation produces a large underestimate of the positron-atom interaction 


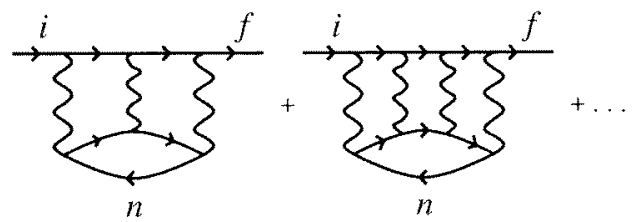

Figure 3. Infinite series of 'ladder' diagrams contributing to the Ps-formation potential. It describes the interaction of the positron (upper line) with one of the atomic electrons.

(see Dzuba et al 1993, Gribakin and King 1994). Physically this is due to the possibility of the positron forming a virtual Ps atom with one of the atomic electrons during the scattering process. In other words one can describe this as tunnelling of the atomic electron to the positron and back. From the many-body-theory point of view this process corresponds to the infinite series of diagrams in figure 3. The contribution of this series is negligible in low-energy electron-atom scattering as it is sign alternating (each successive diagram contains an additional negative energy denominator and a positive electron-electron Coulomb interaction) $\dagger$. In positron-atom scattering (provided the positron energy is less than the Ps-formation threshold) all terms in this series are negative and the sum gives a prominent contribution to the correlation potential. Since the series describes the possibility of virtual Ps formation, the contribution of its sum to the correlation potential will be called the Ps-formation potential, $\Sigma_{E}^{\mathrm{Ps}}$.

The polarization potential, $\Sigma_{E}^{\mathrm{pol}}$ (figure 1 or 2 ) and the Ps-formation potential, $\Sigma_{E}^{\mathrm{Ps}}$ (figure 3) result from the addition of non-intersecting subsets of diagrams. The total correlation potential is found by adding the Ps-formation potential to the polarization potential, so that

$$
\Sigma_{E}=\Sigma_{E}^{\mathrm{pol}}+\Sigma_{E}^{\mathrm{Ps}}
$$

As $\Sigma_{E}^{\mathrm{Ps}}$ is a short-range potential, its addition to $\Sigma_{E}^{\mathrm{pol}}$ does not change the long-range asymptotic behaviour of the correlation potential.

The effect of Ps formation in low-energy positron-atom scattering has been taken into account by various techniques. Amusia et al (1976) reduced the energy denominator of $\Sigma_{E}^{\mathrm{pol}}$ by $6.8 \mathrm{eV}$ in their many-body calculation for He. Basu et al $(1989,1990)$, Hewitt et al (1990), Mitroy (1993), Kernoghan et al (1994) and others have included Ps states in the basis of the close-coupling approximation calculations, Higgins and Burke (1993) included Ps channels in their $R$-matrix calculation (all for hydrogen) and within the manybody formalism the non-local energy-dependent Ps-formation potential was calculated by Gribakin and King (1994). In all of the above cases the effect of virtual Ps formation was essential to describe the positron-atom interaction. These methods all obtained good results for $\mathrm{H}$ or He scattering. The close-coupling approach can be naturally generalized to treat positron scattering by alkali atoms with one valence electron (see, e.g., Hewitt 1993). The advantage of the many-body theory approach over the other methods is that it can describe positron interaction with atoms with many valence electrons in the same way as with $\mathrm{H}$ or He. In the present work we continue the work of Gribakin and King (1994) by calculating the scattering cross sections for noble-gas atoms and also by calculating positron annihilation rates with noble-gas atoms.

$\dagger$ In the vicinity of the ionization threshold these diagrams are more important, since they describe correlations between the two slow electrons in the so-called Wannier regime. 


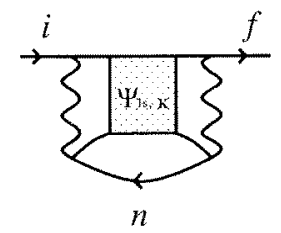

Figure 4. This diagram is used to represent the sum of the ladder diagram series shown in figure 3 (our approximation of the Ps-formation potential).

Firstly, let us represent the series in figure 3 by the single diagram in figure 4, where the shaded rectangle represents the propagation of the interacting electron-positron pair. It is the sum of the series of ladder diagrams with 1,2,3, etc, electron-positron interactions. Unfortunately, it would be quite difficult to sum this series directly. The reason for this is that, the electron-positron pair has bound states, therefore there are poles in the propagation amplitude, which are not present in any of the items of the series. This makes the series non-perturbative. In addition to this, the calculation of the individual diagrams will involve electron and positron states with large orbital momenta. Thus, Bray and Stelbovics (1993) found that in contrast to the electron-hydrogen scattering, it was necessary to include very large $l$ values of up to 15 in their convergent close-coupling calculation of the low-energy positron-hydrogen scattering. The origin of this slow convergence is in the physics of the problem: the partial-wave expansion with respect to the nucleus is used to describe a relatively compact state of the Ps atom formed some distance away from the origin. However, this notion suggested an approximate way of tackling the Ps-formation potential.

Assuming that the Ps formation is dominated by the 1s state, Gribakin and King (1994) suggested the following approximation for $\Sigma_{E}^{\mathrm{Ps}}$ :

$$
\left\langle f\left|\Sigma_{E}^{\mathrm{Ps}}\right| i\right\rangle=\sum_{n} \int \frac{\left\langle f n|V| \tilde{\Psi}_{1 \mathrm{~s}, \boldsymbol{K}}\right\rangle\left\langle\tilde{\Psi}_{1 \mathrm{~s}, \boldsymbol{K}}|V| n i\right\rangle}{E+\varepsilon_{n}-\left(E_{1 \mathrm{~s}}+K^{2} / 4\right)+\mathrm{i} \delta} \frac{\mathrm{d} \boldsymbol{K}}{(2 \pi)^{3}}
$$

where $V=-\left|\boldsymbol{r}-\boldsymbol{r}_{1}\right|^{-1}$ is the electron-positron Coulomb interaction, $\Psi_{1 \mathrm{~s}, K}$ is the wavefunction of the Ps atom in the ground state, moving with momentum $\boldsymbol{K}, E_{1 \mathrm{~s}}+K^{2} / 4$ is the energy of this state, $n$ is the hole state and $\varepsilon_{n}$ is its energy (atomic units are used throughout). The tilde above $\Psi_{1 \mathrm{~s}, K}$ indicates that this wavefunction is orthogonalized to the electron orbitals occupied in the atomic ground state (see below).

The Ps wavefunction $\Psi_{1 \mathrm{~s}, K}$ in (4) can be written as

$$
\Psi_{1 \mathrm{~s}, \boldsymbol{K}}=\varphi_{1 \mathrm{~s}}\left(\boldsymbol{r}-\boldsymbol{r}_{1}\right) \exp (\mathrm{i} \boldsymbol{K} \cdot \boldsymbol{R})
$$

where $\varphi_{1 \mathrm{~s}}\left(\boldsymbol{r}-\boldsymbol{r}_{1}\right)=(8 \pi)^{-1 / 2} \exp \left(-\frac{1}{2}\left|\boldsymbol{r}-\boldsymbol{r}_{1}\right|\right)$ is the hydrogen-like wavefunction of a Ps atom in the ground state and $\boldsymbol{R}=\frac{1}{2}\left(\boldsymbol{r}+\boldsymbol{r}_{1}\right)$ gives the position of the centre of mass of the Ps atom. The approximation of the centre-of-mass motion of the Ps atom by a plane wave is consistent with the neglect of the Ps excitations, since a ground-state Ps atom has zero charge density and does not interact with the ionic field.

As seen in the diagram (figure 4), the shaded rectangle which describes the propagation of the electron-positron pair is constructed from only the excited electron states. Therefore, the Ps wavefunction $\Psi_{1 \mathrm{~s}, K}$ in equation (4) must be orthogonalized to the hole states $v$,

$$
\left|\tilde{\Psi}_{1 \mathrm{~s}, \boldsymbol{K}}\right\rangle=\left(1-\sum_{\nu}|v\rangle\langle\nu|\right)\left|\Psi_{1 \mathrm{~s}, \boldsymbol{K}}\right\rangle
$$

where the sum over $v$ includes all states below the Fermi level.

Above the Ps-formation threshold $\left(E>\left|E_{1 \mathrm{~s}}-\varepsilon_{n}\right|\right)$ the expression (4) acquires an imaginary part, proportional to the square of the matrix element $\left\langle\tilde{\Psi}_{1 \mathrm{~s}, K}|V| n i\right\rangle$ (for $f=i$ ). 
In accordance with the optical theorem, this matrix element should be interpreted as the Ps-formation amplitude in the lowest order. Using equation (6) it can be re-written as

$$
\left\langle\tilde{\Psi}_{1 \mathrm{~s}, \boldsymbol{K}}|V| n i\right\rangle=\left\langle\Psi_{1 \mathrm{~s}, \boldsymbol{K}}|V| n i\right\rangle-\sum_{\nu}\left\langle\Psi_{1 \mathrm{~s}, \boldsymbol{K}} \mid \nu\right\rangle\langle v|V| n i\rangle .
$$

In appendix A, the rearrangement theory approach (see, e.g., Massey and Mohr 1954) is applied to derive the amplitude of the Ps ground-state formation in the positron collision with a many-electron atom. The expression that we have found is identical to the matrix element (7), thus confirming the validity of our approach to the Ps-formation contribution.

The approximation (4) for the diagram in figure 4 obviously neglects virtual Ps formation into states other than the ground state, as well as the interaction of the Ps atom with the hole. We expected the effect of the first of these approximations to be very small for small- to medium-sized atoms. The reason for this is that the creation of the Ps atom is a process which takes place at small positron-atom distances $r \sim r_{\mathrm{a}}$ where valence electrons are localized. Therefore, the amplitude of Ps formation will be roughly proportional to the magnitude of the Ps wavefunction at the origin (or, more precisely, at $r \sim r_{\mathrm{a}}$ ). This would suggest then that the probability of Ps atoms found in the ground state is about eight times larger than that for the $2 \mathrm{~s}$ state. This estimate is supported by the calculations of Ps formation in $1 \mathrm{~s}, 2 \mathrm{~s}$ and $2 \mathrm{p}$ states in positron-hydrogen collisions (see, e.g., Hewitt et al 1990). Indirectly, this physical understanding is supported by the results of the closecoupling calculations of Hewitt et al (1993), which show a progressive increase of the excited Ps formation in the alkali atoms, $\mathrm{Li}, \mathrm{Na}$ and $\mathrm{K}$, with the increase of atomic radius (see also more elaborate close-coupling calculations by McAlinden et al (1994) for Li and $\mathrm{Na}$ and by McAlinden (1996) for K). The radii of all noble-gas atoms are much smaller than even that of $\mathrm{Li}$, so the excited Ps formation should be of much less importance for them.

As well as this, the energy denominator in the diagram (figure 4) describing virtual Ps formation will be smallest for the ground state. We can also refer to the close-coupling calculations of Mitroy (1993) which showed that the addition of the $2 \mathrm{~s}$ and $2 \mathrm{p}$ states of Ps into the expansion produces only a small change in the positron-hydrogen phase shifts. The second approximation, that of neglecting the interaction between the Ps atom and the hole (i.e. the positive ion), should also not produce a large effect, as the static electric field of a Ps atom is zero. One can expect that both of the approximations involved in (4) will produce a small underestimate in the effect that virtual Ps formation has on the positron-atom interaction. Our earlier calculation (Gribakin and King 1994) showed that the Ps-formation potential (4) makes about 20 to $30 \%$ of the total correlation potential (3) and provides good agreement with the precise variational calculations of positron-hydrogen scattering and experimental data for positron-He scattering.

By substituting expression (7) into (4) we obtain an expression for the matrix element of the Ps-formation potential:

$$
\begin{aligned}
\left\langle f\left|\Sigma_{E}^{\mathrm{Ps}}\right| i\right\rangle=\sum_{n} & \int \frac{1}{E+\varepsilon_{n}-E_{1 \mathrm{~s}}-K^{2} / 4+\mathrm{i} \delta} \frac{\mathrm{d} \boldsymbol{K}}{(2 \pi)^{3}} \\
& \times\left[\left\langle f n|V| \Psi_{1 \mathrm{~s}, \boldsymbol{K}}\right\rangle-\sum_{\nu}\langle f n|V| v\rangle\left\langle v \mid \Psi_{1 \mathrm{~s}, \boldsymbol{K}}\right\rangle\right] \\
& \times\left[\left\langle\Psi_{1 \mathrm{~s}, \boldsymbol{K}}|V| n i\right\rangle-\sum_{\nu^{\prime}}\left\langle\Psi_{1 \mathrm{~s}, \boldsymbol{K}} \mid \nu^{\prime}\right\rangle\left\langle v^{\prime}|V| n i\right\rangle\right] .
\end{aligned}
$$

The evaluation of equation (8) is done by expanding the Ps wavefunctions and the Coulomb interactions in terms of spherical harmonics with respect to the nucleus. The integrals over 
all angular variables can be done analytically and the radial integrals and the integral over $\mathrm{d} K$ are evaluated numerically. The corresponding formulae are given in appendix $\mathrm{B}$. We should note that it is sufficient to take into account only one outer atomic subshell $n$, which has the largest radius and smallest binding energy $\left|\varepsilon_{n}\right|$ (e.g. the $n$ p subshell for noble-gas atoms). However, the orthogonalization sum over $v$ must be performed over two or three subshells (i.e. $n \mathrm{p}, n \mathrm{~s}$ and $(n-1) \mathrm{d})$. Also, the Ps-formation potential can be calculated in the coordinate representation as $\Sigma_{E l}^{\mathrm{Ps}}\left(r, r^{\prime}\right)$ for each positron partial wave $l$. It is then added to the polarization potential $\Sigma_{E l}^{\mathrm{pol}}\left(r, r^{\prime}\right)$ (figure $\left.2(c)\right)$ and used to calculate the positron radial wavefunction from equation (1). The scattering phase shifts are found from the asymptotic behaviour of the wavefunction.

\section{Results for positron-noble-gas atoms elastic scattering}

In order to highlight the role of the Ps-formation potential we compare below the results obtained with $\Sigma_{E}=\Sigma_{E}^{\text {pol }}$ with those produced by the 'full' correlation potential (3). The positron scattering lengths of $\mathrm{Ne}, \mathrm{Ar}, \mathrm{Kr}$ and $\mathrm{Xe}$ are presented in table 1. The total scattering cross sections shown in figure 5 as a function of the positron momentum $p$ were found by summing the partial cross sections (for low-energy scattering, only the $\mathrm{s}, \mathrm{p}$ and $\mathrm{d}$ partial waves need to be calculated). Also shown in this figure are the cross sections obtained for positrons scattered by the static potential of the Hartree-Fock atomic ground state. This potential is totally repulsive and the corresponding cross sections do not bear any resemblance to the true ones.

Table 1. Scattering lengths and virtual level energies for positrons scattered from $\mathrm{Ne}, \mathrm{Ar}, \mathrm{Kr}$ and $\mathrm{Xe}$.

\begin{tabular}{lcrcl}
\hline Atom & $\mathrm{Ne}$ & $\mathrm{Ar}$ & $\mathrm{Kr}$ & $\mathrm{Xe}$ \\
\hline$a(\mathrm{au})$ & -0.43 & -3.9 & -9.1 & $\approx-100$ \\
$\varepsilon(\mathrm{eV})$ & - & 0.9 & 0.16 & $\approx 0.001$ \\
\hline
\end{tabular}

If the correlation potential includes only the polarization potential, the cross section is given by the long-broken curve. One can see from figure 5 that they qualitatively reproduce the experimental cross sections, e.g. display the Ramsauer-Townsend minimum (or its traces, in heavier atoms) which is due to the scattering length becoming negative and the s-wave phase shift passing through zero at small energy. The fact that the scattering lengths are negative means that the attraction due to the polarization of the atom by the positron field effectively overcomes the repulsion experienced by the positron in the static atomic potential.

If the correlation potential includes both the polarization potential and the Ps-formation potential the cross section is given by the full curve. It is clear that the Ps-formation potential produces a significant change in the cross sections and greatly improves the agreement of our calculations with the experimental data points. It also makes the scattering lengths $a$ for $\mathrm{Ar}, \mathrm{Kr}$ and $\mathrm{Xe}$ much greater than the corresponding atomic radii, which means the existence of positron-atom virtual levels at $\varepsilon \simeq \hbar^{2} / 2 m a^{2}$ (see table 1). Such virtual levels have important consequences for the positron-atom annihilation problem. For Xe the huge magnitude of the scattering length might have a further implication that one can observe true binary positron-Xe collisions only at densities well below 1 Amagat, when the mean interatomic distance $\bar{r}$ becomes greater than the scattering length $a$. Otherwise, for low- 

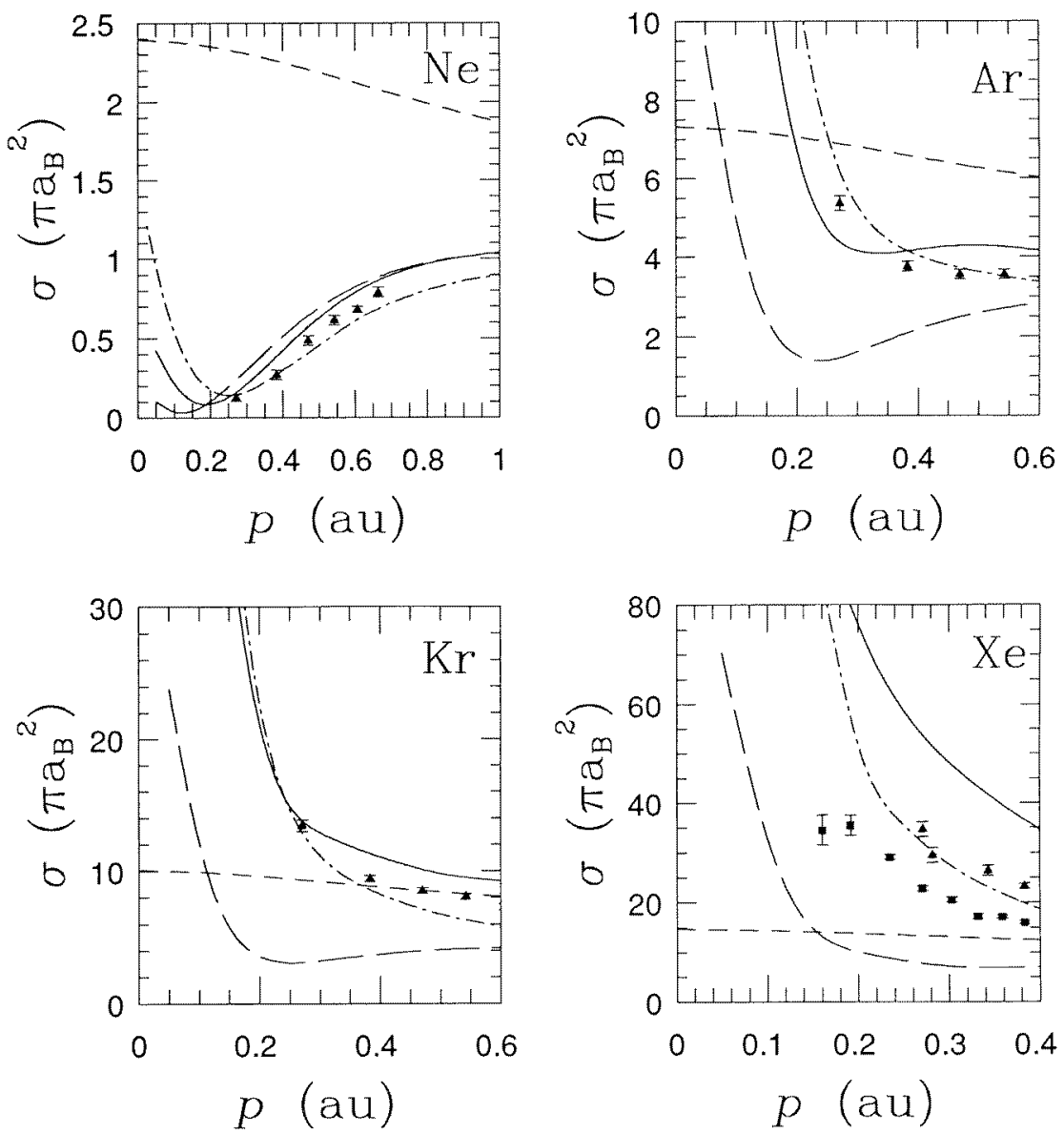

Figure 5. Comparison of the elastic scattering cross sections for $\mathrm{Ne}, \mathrm{Ar}, \mathrm{Kr}$ and $\mathrm{Xe}$. Theoretical: - , present result $\left(\Sigma_{E}^{\mathrm{pol}}+\Sigma_{E}^{\mathrm{Ps}}\right) ;--\_$, calculations without the Ps-formation contribution $\left(\Sigma_{E}^{\mathrm{pol}}\right.$ only); ---, Hartree-Fock (static atomic potential); - - - , results of McEachran et al (1978, 1979, 1980). Experimental: $\mathbf{\square}$, Sinapius et al (1980), \, Dababneh et al (1980).

energy positrons, $p \bar{r} \lesssim 1$, the effects of multiple scattering are important if $\bar{r} \sim a$. They can be described in other words as clustering, since the interaction of the positron with several nearby Xe atoms becomes important (see section 4.3).

We must stress that the accuracy of the approximation adopted for the correlation potential does not allow us to consider either the scattering length or the energy of the virtual level firmly established for Xe. For example, when we used in (3) the polarization potential without the electron-hole interaction and screening (in figure $1(a)$ ), the scattering length would become positive and a bound positron-Xe state with $-0.005 \mathrm{eV}$ energy would emerge. We also checked that a 5\% reduction of the correlation potential would then eliminate the binding. However, this would produce very little difference for the elastic cross section above several meV and the estimate $|a| \sim 100$ au would remain valid in any case. Note that recently we have examined several atoms $(\mathrm{Mg}, \mathrm{Zn}, \mathrm{Cd}$ and $\mathrm{Hg}$ ) which have larger dipole polarizabilities and smaller ionization potentials than those of Xe (Dzuba et al 1995). Our calculations, which are similar to the present ones, show that these atoms can 
form true bound states with positrons, with binding energies ranging from 0.87 to $0.045 \mathrm{eV}$. The large strength of the binding secures the result against possible uncertainties in the correlation potential. Some evidence has been presented that there are other atoms which could form bound states with positrons. Comparison of the calculated (Gribakin and King 1996) and measured (Jiang et al 1996) cross sections for positrons on Mg indirectly supports the existence of the bound state in this system.

The chain curves in figure 5 are the results of McEachran et al (1978b, 1979, 1980). These cross sections are produced using the polarized orbital approximation, in which the positron-atom system is treated using the adiabatic approximation and the monopole polarization contribution is neglected. They do not explicitly include the effects of virtual Ps formation. With respect to our calculation, this approximation corresponds to omitting the intermediate energy of the positron in the diagram in figure $2(c)$. The positron is a light particle, hence the adiabatic approximation produces a noticeable overestimate of the positron-atom interaction. It appears that in this energy range the incurred overestimation has quite accurately compensated for the neglect of virtual Ps formation.

\section{Positron annihilation rates in noble gases}

\subsection{Many-body theory calculations}

The annihilation rate $\lambda$, of a positron in a gas of density $n$, may be expressed in terms of the effective number of electrons $\left(Z_{\text {eff }}\right)$ of the atom (see, e.g., Akhiezer and Berestetskii 1965) by the expression

$$
\lambda=\pi r_{0}^{2} c n Z_{\text {eff }}
$$

where $r_{0}$ is the classical radius of the electron and $c$ is the speed of light. At low densities, where there are no clusters and only binary positron-atom collisions contribute, the $Z_{\text {eff }}$ is independent of density and characterizes the positron-atom system. Definition (9) means that $Z_{\text {eff }}$ is the ratio of the positron annihilation cross section of the atom to the annihilation cross section of a free electron in the Born approximation. Accordingly, the expression for $Z_{\text {eff }}$ of an atom can be written as

$$
Z_{\text {eff }}=\sum_{i=1}^{N} \int\left|\Psi\left(\boldsymbol{r}_{1}, \boldsymbol{r}_{2}, \ldots, \boldsymbol{r}_{N}, \boldsymbol{X}\right)\right|^{2} \delta\left(\boldsymbol{r}_{i}-\boldsymbol{X}\right) \mathrm{d} \boldsymbol{r}_{1} \mathrm{~d} \boldsymbol{r}_{2} \ldots \mathrm{d} \boldsymbol{r}_{N} \mathrm{~d} \boldsymbol{X}
$$

where $\Psi\left(\boldsymbol{r}_{1}, \boldsymbol{r}_{2}, \ldots, \boldsymbol{r}_{N}, \boldsymbol{X}\right)$ is the full $(N+1)$-particle wavefunction, $\boldsymbol{X}$ is the positron coordinate and the wavefunction is normalized so that the asymptotic form at $|\boldsymbol{X}| \gg r_{\mathrm{a}}$ is $\Psi_{0}\left(\boldsymbol{r}_{1}, \boldsymbol{r}_{2}, \ldots, \boldsymbol{r}_{N}\right) \exp (\mathrm{i} \boldsymbol{p} \cdot \boldsymbol{X}), \boldsymbol{p}$ being the incoming positron momentum. Using this normalization the $Z_{\text {eff }}$ value of an electron in the Born approximation is equal to one. It is worth noting that formally expression (10) has the structure of a matrix element of the $\sum_{i} \delta\left(\boldsymbol{r}_{i}-\boldsymbol{X}\right)$ operator (although $Z_{\text {eff }}$ itself is rather a cross section). This enables one to develop a diagrammatic technique for calculating $Z_{\text {eff }}$, similar to that used, e.g., for the scattering amplitude (Dzuba et al 1993, see below).

As one might expect from the asymptotic form of the wavefunction, $Z_{\text {eff }}$ is a function of the positron momentum $p$. The experimental determination of $Z_{\text {eff }}$ effectively measures the thermal average of $Z_{\mathrm{eff}}(p)$ (provided the positrons are thermalized). If $Z_{\mathrm{eff}}(p)$ is flat at thermal energies $(p \approx 0.045)$ then one can calculate $Z_{\text {eff }}$ at this value of $p$. Otherwise, as we will see in the case of $\mathrm{Xe}$, we must calculate $Z_{\mathrm{eff}}(p)$ and convolve this with the Maxwellian distribution. In any case, for such small momenta only the s-wave component of the 
Table 2. The $Z_{\text {eff }}$ values for hydrogen and the noble gases. a, b and $\mathrm{c}$ are the contributions to the $Z_{\text {eff }}$ value of the diagram in figure 6 , where the positron wavefunction is calculated in the static potential of the Hartree-Fock atomic ground state, with the correlation potential $\Sigma_{E}=\Sigma_{E}^{\mathrm{pol}}$ and with $\Sigma_{E}=\Sigma_{E}^{\mathrm{pol}}+\Sigma_{E}^{\mathrm{Ps}}$, respectively. Contributions of other diagrams obtained for the positron wavefunction calculated with $\Sigma_{E}=\Sigma_{E}^{\mathrm{pol}}+\Sigma_{E}^{\mathrm{Ps}}$ : d, diagram in figure 9; e, diagram in figure 10; $\mathrm{f}$, the total $Z_{\text {eff }}$ value from our many-body calculation, including estimates of uncalculated diagrams. $\mathrm{g}$, the $Z_{\text {eff }}$ value resulting from the simplistic inclusion of the Sommerfeld factor. h, the accepted $Z_{\text {eff }}$ values (for hydrogen, Bhatia et al (1974), for noble gases, Coleman et al (1975)). i, experimental values obtained in the positron trap, Iwata et al (1995) for Ar and $\mathrm{Kr}$ and Murphy and Surko (1990) for Xe.

\begin{tabular}{llllllc}
\hline Atom & $\mathrm{H}$ & $\mathrm{He}$ & $\mathrm{Ne}$ & $\mathrm{Ar}$ & $\mathrm{Kr}$ & $\mathrm{Xe}$ \\
\hline $\mathrm{a}$ & 0.41 & 0.69 & 0.97 & 0.75 & 0.70 & 0.65 \\
$\mathrm{~b}$ & 0.94 & 1.17 & 2.03 & 3.90 & 5.55 & 10.3 \\
$\mathrm{c}$ & 1.16 & 1.31 & 2.22 & 5.1 & 11.3 & 73.6 \\
$\mathrm{~d}$ & 1.45 & 1.05 & 1.3 & 5.0 & 11.7 & 83.3 \\
$\mathrm{e}$ & 0.05 & 0.003 & 0.007 & 0.1 & 0.5 & 6.1 \\
$\mathrm{f}$ & 3.9 & 2.7 & 4.0 & 13.6 & 33.8 & 250 \\
$\mathrm{~g}$ & 5.7 & 4.4 & 5.7 & 18.7 & 45.3 & 340 \\
$\mathrm{~h}$ & 7.5 & 3.94 & 6 & 27 & 65 & $<320$ \\
$\mathrm{i}$ & - & - & - & 33.8 & 90.1 & 401 \\
\hline
\end{tabular}

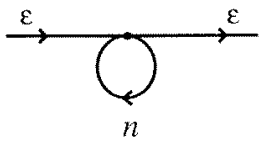

Figure 6. Diagrammatic representation of $Z_{\text {eff }}^{(0)}$, the lowest-order contribution to $Z_{\text {eff }}$. The positron wavefunction $\varepsilon$ can be calculated either in the static atomic potential of the Hartree-Fock ground state or with the correlation potential $\Sigma_{E}$ taken into account.

positron wavefunction has to be taken into account, since higher-partial-wave contributions are suppressed as $p^{2 l}$.

In the Hartree-Fock approximation $\Psi\left(\boldsymbol{r}_{1}, \boldsymbol{r}_{2}, \ldots, \boldsymbol{r}_{n}, \boldsymbol{X}\right)$ is the product of the positron wavefunction $\psi(\boldsymbol{X})$ (calculated in the static field of the atom) and the wavefunction of the electrons $\Psi_{0}\left(\boldsymbol{r}_{1}, \boldsymbol{r}_{2}, \ldots, \boldsymbol{r}_{N}\right)$ which is the antisymmetrized product of the single-particle orbitals $\psi_{n}(\boldsymbol{r})$ of the atom. Therefore, in the Hartree-Fock approximation equation (10) becomes

$$
Z_{\text {eff }}^{(0)}=\sum_{n=1}^{N} \int\left|\psi_{n}(\boldsymbol{r})\right|^{2}|\psi(\boldsymbol{X})|^{2} \delta(\boldsymbol{r}-\boldsymbol{X}) \mathrm{d} \boldsymbol{r} \mathrm{d} \boldsymbol{X} .
$$

This may be represented graphically by the diagram in figure 6 . Figure 6 is similar to a many-body scattering diagram and is calculated using the same rules. The $Z_{\text {eff }}$ values found in this way are presented in row a of table 2. Note that in this section we consider $\mathrm{H}$ and $\mathrm{He}$, as well as the heavier noble-gas atoms. The problem of positron scattering by these two atoms has been considered within the present many-body approach earlier (Gribakin and King 1994). The experimental values of $Z_{\text {eff }}$ are between 5 and 300 times higher than these values. This difference between the experimental values and the Hartree-Fock results gives an indication of the important role that correlation effects play in the annihilation of positrons with atoms.

The first improvement to this approximation is to use a better positron wavefunction $\psi(\boldsymbol{X})$, the one found from the Dyson equation, equation (1) with the positron-atom correlation potential taken into account. In principle, if $\Sigma_{E}$ were known exactly, this wavefunction would be the best single-particle positron wavefunction with exact positronatom phase shifts. Rows b and c show the value of $Z_{\mathrm{eff}}^{(0)}$ when the correlation potential $\Sigma_{E}$ 
is equal to $\Sigma_{E}^{\mathrm{pol}}$ and $\Sigma_{E}^{\mathrm{pol}}+\Sigma_{E}^{\mathrm{Ps}}$, respectively. Note that in the latter case the phase shifts of the positron wavefunction produced the cross sections in good agreement with experiment, figure 5. We can see from table 2 that the correlation effects alter the positron wavefunction so as to produce a huge increase of $Z_{\text {eff }}^{(0)}$ and that Ps formation is responsible for a large part of these correlations. In Xe, the correlations increase $Z_{\text {eff }}{ }^{(0)}$ by a factor of 100 . However, the experimental values of $Z_{\text {eff }}$ are typically four times larger than the $Z_{\text {eff }}{ }^{(0)}$ shown in row c.

Although, from the scattering cross sections, the positron wavefunction appears to be quite accurate, $Z_{\mathrm{eff}}^{(0)}$ is strongly deficient for the description of positron annihilation. The difference in the calculation of rows $\mathrm{a}, \mathrm{b}$ and $\mathrm{c}$ in table 2 , is the corrections to the positron wavefunction, caused by the positron-atom correlation potential. That is, the corrections to the 'ends' in figure 6. To calculate the annihilation rate properly we must take into account the corrections to the vertex (see Dzuba et al 1993).

In other words, while improving the positron wavefunction we have so far approximated the total wavefunction of the system as $\psi(\boldsymbol{X})$ times the Hartree-Fock atomic ground-state wavefunction. Now we have to include corrections to the positron-atom wavefunction. From the physical point of view the most important corrections arise from the Coulomb interaction of the annihilating electron-positron pair. This interaction determines the exact magnitude of the wavefunction at small (zero) electron-positron separations. The wavefunction with such corrections is represented by the sum of diagrams in figure 7 . The first term is the product of the positron wavefunction and the ground-state electron wavefunction (the ground-state electron wavefunction is not shown in many-body diagrams). The square of this diagram gives the contribution to the $Z_{\text {eff }}$ value that is shown in figure 6 , equation (11). The other terms are electron-positron correlation corrections and as such are not trivial to calculate $\dagger$.

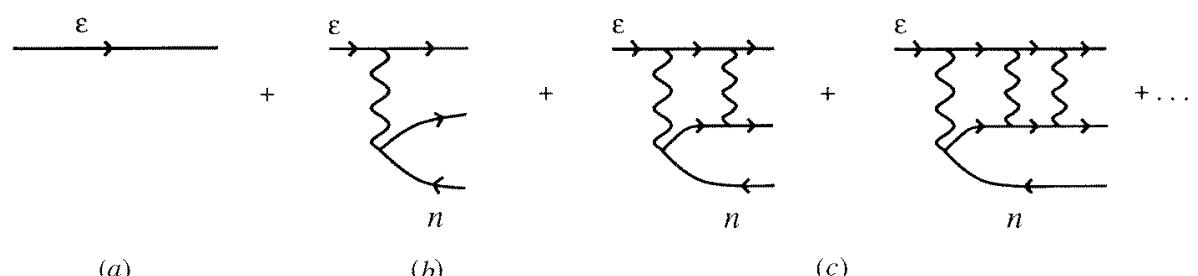

Figure 7. Diagrammatic representation of the wavefunction of the positron-atom system diagrams. Diagrams $(b)$ and $(c)$ are the corrections due to the electron-positron Coulomb interaction. They are most essential for the correct calculation of the wavefunction at small electron-positron separations.

Figure 8 gives the approximation that we have actually used in the calculation of the annihilation rates. We have again used virtual Ps formation to describe the infinite series of ladder diagrams that correct the wavefunction at zero positron-electron separation. Once again in the numerical calculations we include only the Ps 1 s state and neglect the interaction between the Ps atom and the ionic residue. Using the approximation in figure 8 , the $Z_{\text {eff }}$

$\dagger$ One can consider more complicated correlation corrections involving excitations of more than one atomic electron. However, the other electrons will just be spectators during the annihilation of the positron with the first electron and such corrections should be of less importance. 


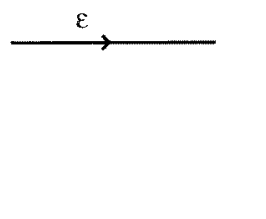

(a)

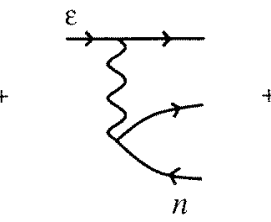

(b)

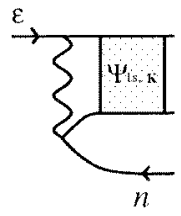

(c)

Figure 8. Approximation that we have used for the positron-atom wavefunction in the annihilation rate calculations. Diagram $(c)$ is meant to account for the electron-positron annihilation in the virtual Ps state.

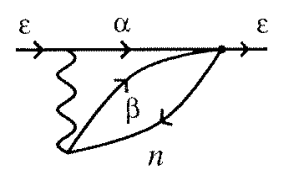

(a)

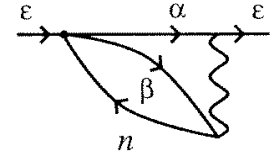

(b)

Figure 9. Diagrammatic representation of the $Z_{\mathrm{eff}}^{(2 a b)}$ contribution.

value of an atom may now be written as

$Z_{\text {eff }}=\sum_{i=1}^{N} \int\left(\left\langle a\left|+\left\langle b|+\langle c|) \delta\left(\boldsymbol{r}_{i}-\boldsymbol{X}\right)(|a\rangle+|b\rangle+|c\rangle) \mathrm{d} \boldsymbol{r}_{1} \ldots \mathrm{d} \boldsymbol{r}_{n} \mathrm{~d} \boldsymbol{X}\right.\right.\right.\right.$

where $|a\rangle,|b\rangle$ and $|c\rangle$ are the symbolic notations for the contributions of the three diagrams in figure 8. One can see from this equation that there will be three diagonal contributions, $Z_{\text {eff }}{ }^{\left(a^{2}\right)}, Z_{\text {eff }}^{\left(b^{2}\right)}$ and $Z_{\text {eff }}{ }^{\left(c^{2}\right)}$ (the first of them is equivalent to $Z_{\text {eff }}{ }^{(0)}$, equation (11), figure 6) and three non-diagonal terms, $Z_{\text {eff }}{ }^{(2 a b)}, Z_{\text {eff }}^{(2 a c)}$ and $Z_{\text {eff }}{ }^{(2 b c)}$.

The first-order correction to the zeroth-order diagram (figure 6) results from the product of the $a$ and $b$ terms in equation (12) and is given by the two equal-valued diagrams shown in figure 9. The sum of these two diagrams can be written as

$$
\begin{aligned}
Z_{\mathrm{eff}}^{(2 a b)} & \equiv \sum_{i=1}^{N}\left\langle a\left|\delta\left(\boldsymbol{r}_{i}-\boldsymbol{X}\right)\right| b\right\rangle+\sum_{i=1}^{N}\left\langle b\left|\delta\left(\boldsymbol{r}_{i}-\boldsymbol{X}\right)\right| a\right\rangle \\
& =-2 \sum_{\alpha \beta n} \frac{\langle\varepsilon n|\delta(\boldsymbol{r}-\boldsymbol{X})| \beta \alpha\rangle\langle\alpha \beta|V| n \varepsilon\rangle}{E-\varepsilon_{\alpha}-\varepsilon_{\beta}+\varepsilon_{n}} .
\end{aligned}
$$

This expression is very similar to the second-order scattering diagram shown in figure 1(a), with one of the Coulomb interactions replaced by the $\delta$-function. The contribution of equation (13) to the $Z_{\text {eff }}$ value, where the positron wavefunction was calculated with the correlation potential $\Sigma_{E}=\Sigma_{E}^{\text {pol }}+\Sigma_{E}^{\text {Ps }}$, is shown in row d of table 2. Their addition to $Z_{\text {eff }}{ }^{\left(a^{2}\right)} \equiv Z_{\text {eff }}^{(0)}$ (row c) almost doubles the magnitude of $Z_{\text {eff. These diagrams were }}$ calculated in Dzuba et al (1993), where they were also found to be important. The fact that this contribution is comparable to the zeroth-order diagram, is indicative that the vertex correction series will be at best slowly convergent.

We must note that the contribution (13) is analogous to the first-order correction to $Z_{\text {eff }}$ included in the polarized-orbital calculations of McEachran et al (1977). In spite of the absence of higher-order corrections they obtained surprisingly good values of $Z_{\text {eff }}$ for noble-gas atoms (see section 2). In contrast to the many-body theory, their approach includes 
an adiabatic treatment of the positron that corresponds to the neglect of the intermediate positron energy $\varepsilon_{\alpha}$ in the correlation-potential diagrams (figure $1(a)$ ), and in the annihilation diagrams (figure 9). This obviously produces a considerable overestimate of the first-order correction (13), and makes for the absence of the higher-order corrections (as well as for the neglect of the Ps-formation contribution in the scattering problem).

It is quite easy to calculate the contribution to $Z_{\text {eff }}$ from the squared Ps-formation amplitude (figure $8(c)$ ). The corresponding annihilation diagram is shown in figure 10 and the analytic expression for it is

$$
\begin{aligned}
Z_{\mathrm{eff}}^{\left(c^{2}\right)} & \equiv \sum_{i=1}^{N}\left\langle c\left|\delta\left(\boldsymbol{r}_{i}-\boldsymbol{X}\right)\right| c\right\rangle \\
& =\int \frac{\left\langle\varepsilon n|V| \Psi_{1 \mathrm{~s}, \boldsymbol{K}}\right\rangle\left|\varphi_{1 \mathrm{~s}}(0)\right|^{2}\left\langle\Psi_{1 \mathrm{~s}, \boldsymbol{K}}|V| n \varepsilon\right\rangle}{\left(E+\varepsilon_{n}-K^{2} / 4-E_{1 \mathrm{~s}}\right)^{2}} \frac{\mathrm{d} \boldsymbol{K}}{(2 \pi)^{3}} \\
& =-\left|\varphi_{1 \mathrm{~s}}(0)\right|^{2} \frac{\partial}{\partial E}\left\langle\varepsilon\left|\Sigma_{E}^{\mathrm{Ps}}\right| \varepsilon\right\rangle
\end{aligned}
$$

where $\left|\varphi_{1 \mathrm{~s}}(0)\right|^{2}=1 / 8 \pi$ is the density of the ground-state Ps state at the origin. The contribution to $Z_{\text {eff }}$ from this diagram is shown in row e of table 2 and appears to be quite small.

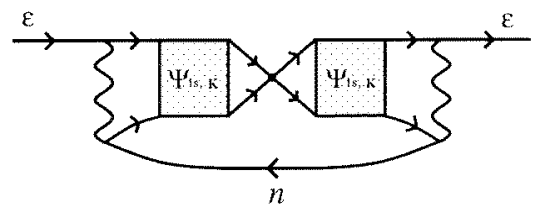

Figure 10. Diagrammatic representation of the $Z_{\text {eff }}^{\left(c^{2}\right)}$ contribution.

We have now calculated three of the six terms that need to be evaluated (see equation (12)). To approximate the rest of the terms, we assumed that the annihilation diagrams are proportional to the contributions to the wavefunction ( $a, b$ or $c$ ) which produce them. Then, after calculating the $a^{2}-, 2 a b$ - and $c^{2}$-type contributions, the rest of them are estimated in a simple way. For example,

$$
\begin{aligned}
Z_{\mathrm{eff}}^{\left(b^{2}\right)} & \simeq C b^{2} \\
& =C\left(\frac{2 a b}{2 \sqrt{a^{2}}}\right)^{2} \\
& \simeq\left(Z_{\mathrm{eff}}^{(2 a b)} / 2\right)^{2} / Z_{\mathrm{eff}}^{\left(a^{2}\right)} .
\end{aligned}
$$

The remaining terms (' $2 a c$ ' and ' $2 b c$ ') are approximated in the same manner and the total of all six terms is shown in row $\mathrm{f}$ of table 2.

The inclusion of the Ps-formation potential in the scattering problem and the Psformation contribution in $Z_{\text {eff }}$ has improved the agreement between the experimental and our theoretical values of $Z_{\text {eff }}$. However, despite the fact that our calculations of the scattering cross sections agree well with the experimental curves, there is still a significant discrepancy between the accepted experimental values and our values of $Z_{\text {eff }}$. We believe that the origin of this difference is in the approximate way in which we treat the electron-positron ladder diagram series. Why is it then that the same ground-state Ps approximation of this series is quite good for the scattering problem and rather poor for the annihilation problem? 
Compare the ladder-diagram series for the correlation potential (scattering problem, figure 3) and the wavefunction to be used for calculating the annihilation rate (figure 7). In the former case the electron returns back to fill its parent hole state and the typical distances occupied by the interacting positron and electron are comparable to the Ps or atomic radii (1-2 au). Of course, bringing the positron and electron closer would result in a stronger Coulomb attraction between them. However, this process involves greater kinetic energies and consequently, produces large energy denominators in the diagrams. Therefore, excitation of the Ps continuum gives a small contribution because this is a highly virtual process (note that the double continuum of the noninteracting positron and electron is already included in figure $1(a)$ ). We also remind the reader that excitations of the Ps discrete states above the 1s state are suppressed, since they are too diffuse (see section 2).

In the annihilation problem the value of $Z_{\text {eff }}$ is determined by the wavefunction at zero positron-electron separation, i.e. the interacting positron and electron are necessarily brought together by the $\delta$-function vertex (like in figure 9 or 10). This enhances the role of the continuum states distorted by the positron-electron Coulomb attraction. Therefore, the approximation that the Ps is only formed in the ground state, can produce a substantial underestimate in the calculation of the annihilation rates. In other words, approximating the two-particle (positron and electron) Green function by the freely propagating Ps in the ground state is quite inaccurate when the two particles end up at the same point in space where they annihilate. One must find a better way of summing the ladder-diagram series, or approximating the sum, for this problem.

\subsection{Other approximations of the higher-order terms}

In order to understand the extreme significance of the vertex corrections $\left(Z_{\text {eff }}^{(2 a b)}, Z_{\text {eff }}^{\left(b^{2}\right)}\right.$, etc), we would like to make a semi-quantitative estimate. If we consider a positron of momentum $k$ scattering from a fixed unit point charge, the Coulomb interaction enhances the wavefunction of the positron near the charge by the Sommerfeld factor $S_{\infty}(k)$ (see, e.g., Landau and Lifshitz 1965):

$$
\begin{aligned}
S_{\infty}(k) & =\frac{\left|\psi_{k}(0)\right|^{2}}{\left|\psi_{k}(\infty)\right|^{2}} \\
& =\frac{2 \pi}{k\left(1-\mathrm{e}^{-2 \pi / k}\right)} \\
& \simeq \frac{2 \pi}{k} \quad(k \ll 2 \pi) .
\end{aligned}
$$

This factor corrects the Born-approximation value $\left(Z_{\text {eff }}=1\right)$ for positron-electron annihilation. Equation (16) was derived by assuming that the positron experiences the Coulomb interaction from infinity. In the case of a positron interacting with an atomic electron, the Coulomb interaction between them is 'screened' $\dagger$ by the nucleus, until the positron comes close to the atom. We have found that this can be described by a modified Sommerfeld factor

$$
S_{R}(k) \simeq \frac{2 \pi}{\sqrt{k^{2}+2 / R}}
$$

where $R$ is the distance beyond which the electron-positron Coulomb interaction can be neglected. It has the magnitude of the atomic radius.

$\dagger$ As the nucleus is heavy it does not screen the Coulomb interaction in the usual sense, but at large positron-atom distances the nuclear charge does cancel the Coulomb interaction of the positron with atomic electrons. 
The $k$ in the above equations is the relative momentum of the positron-electron pair. We are considering the annihilation of thermal positrons at about $300 \mathrm{~K}$, so the positron momentum is very small. The relative momentum of the positron and atomic electron $k$, is therefore determined by the momentum distribution of the bound electrons. Thus, we must integrate the Coulomb enhancement factor over $\boldsymbol{k}$ with the weight given by the squared Fourier transform of the atomic electron wavefunction. This 'average' Coulomb enhancement of the positron wavefunction at each of the atomic electrons $\left(S_{\mathrm{at}}\right)$ may be written as

$$
S_{\text {at }}=\int|a(\boldsymbol{k})|^{2} S(k) \frac{\mathrm{d} \boldsymbol{k}}{(2 \pi)^{3}}
$$

where $a(\boldsymbol{k})$ is the Fourier transform of the electron wavefunction. $S(k)$ is either $S_{\infty}(k)$ or $S_{R}(k)$. The positron does not penetrate deep into the atom and the annihilation will mainly take place with one of the valence electrons. So, one can use the Fourier transform of the valence electron wavefunction for $a(\boldsymbol{k})$. We used the analytic electron wavefunctions from Ratsig and Smirnov (1986) to calculate $S_{\text {at }}$ and took the 'cut-off' radius $R=1 / I$ equal to the classical turning point for the atomic valence electrons in the $1 / r$ ionic field. Note that if the momenta of atomic electrons were very large $(k \gg 1, S(k) \rightarrow 1)$, the atomic enhancement factor (18) would be equal to 1 , which simply follows from the normalization of $a(\boldsymbol{k})$.

According to the derivation, $S_{\text {at }}$ is the coefficient which accounts for the enhancement of $Z_{\text {eff }}^{(0)}$ due to the Coulomb interaction between the annihilating positron and electron. We should remind the reader that $Z_{\text {eff }}{ }^{(0)}$, equation (11), contains the product of the positron and electron wavefunctions and, as such, does not include the electron-positron pair correlations. The two approximations of $S_{\text {at }}$ are plotted in figure 11 along with the factor required to bring our $Z_{\text {eff }}{ }^{(0)}$ values into agreement with the accepted experimental values, $Z_{\text {exp }} / Z_{\text {eff }}{ }^{(0)}$ (where $Z_{\text {exp }}$ and $Z_{\text {eff }}{ }^{(0)}$ are taken from rows $\mathrm{h}$ and $\mathrm{c}$ of table 2 , respectively). As one would expect, the value of $S_{\text {at }}$, when $S_{\infty}$ is used, overestimates the Coulomb enhancement. When $S_{R}$ is used, a better approximation of the Coulomb enhancement is achieved. This means that if one has found an accurate solution of the scattering problem and obtained a good single-particle positron wavefunction, the latter can be used to calculate $Z_{\text {eff }}^{(0)}$. Then, after multiplying $Z_{\text {eff }}{ }^{(0)}$ by $S_{\text {at }}$, very reasonable values of the annihilation rate are obtained.

We have seen that the first-order correction $Z_{\mathrm{eff}}^{(2 a b)}$ has roughly the same magnitude as $Z_{\text {eff }}{ }^{(0)}$. This means that the whole ladder-diagram series should be summed. Instead of using the ground-state Ps approximation, we can evaluate the result by assuming that the relevant diagrams form a geometric series. Each successive term of the series in figure 8 has an extra Coulomb interaction. Suppose that it effectively multiplies the diagram by a factor of $q$. Then we can write the total as $a+a q+a q^{2}+\cdots$. Summing this infinite series we obtain instead of equation (12),

$$
\begin{aligned}
Z_{\text {eff }} & =Z_{\text {eff }}^{\left(a^{2}\right)} \frac{1}{(1-q)^{2}} \\
& =Z_{\text {eff }}^{(0)} S_{q} .
\end{aligned}
$$

The value of $q$ can be obtained from the relative magnitude of the first-order correction, $q \approx Z_{\text {eff }}^{(0)} / 2 Z_{\text {eff }}^{(2 a b)}$. Using the values from table 2 , we can obtain $q$ for each of the atoms presented. The factor $S_{q}=(1-q)^{-2}$ is plotted in figure 11. This approximation has also produced reasonable agreement with the enhancement factors required by the experimental values.

Both of these crude approximations produce similar estimates for the infinite series of diagrams that correct the electron-positron annihilation vertex. The fact that all the curves in 


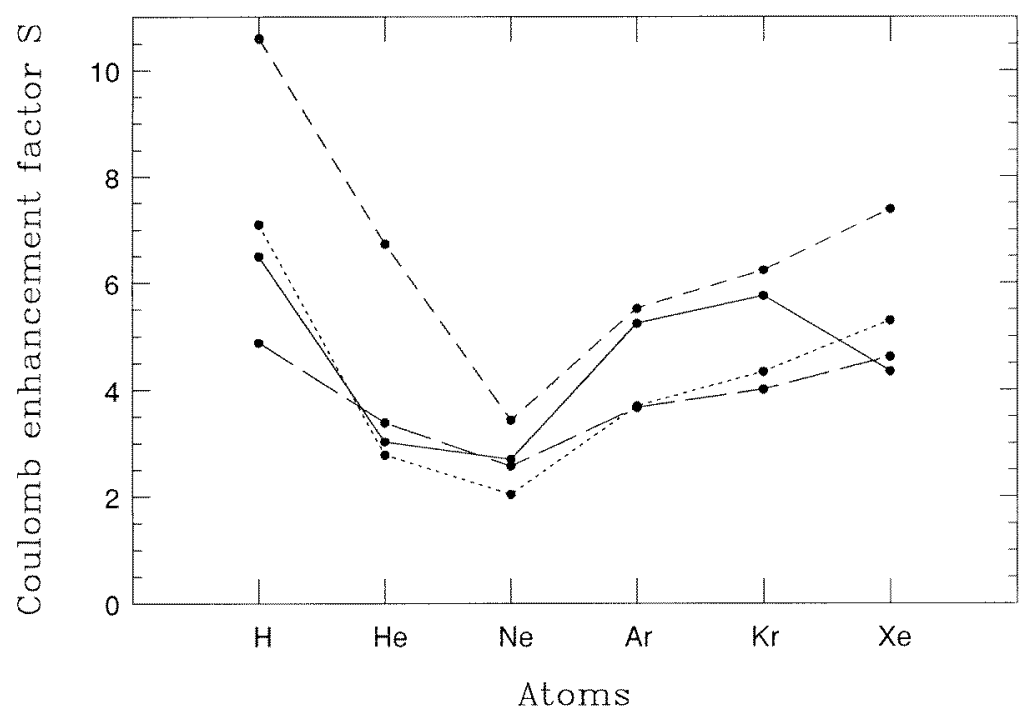

Figure 11. Approximations of the higher-order annihilation diagrams. - - the ratio of the experimental values to our $Z_{\text {eff }}^{(0)}$ results; - - , the $S_{\text {at }}$ factor calculated using $S_{\infty} ;-\ldots$, the $S_{\text {at }}$ factor calculated using $S_{R} ;-\cdots, S_{q}$.

figure 11 have the same qualitative features as the full curve suggests that our understanding of the problem is correct. However, the lack of a rigorous treatment of this series in our many-body calculations is the reason that we are unable to reproduce the experimental values.

\subsection{Role of the positron-atom virtual levels: the Xe annihilation rate}

The behaviour of $Z_{\text {eff }}$ versus $p$ for the lighter noble-gas atoms is quite flat in the range of thermal positron momenta. Therefore, it is sufficient to calculate the value of $Z_{\text {eff }}$ for a positron with thermal energy $(p \approx 0.045)$. The dependence of $Z_{\text {eff }}$ on positron momentum for $\mathrm{Xe}$ is very strong and, therefore, we must average $Z_{\text {eff }}(p)$ over the Maxwellian distribution $\dagger$. What makes Xe so different from the other noble-gas atoms? It was understood in Dzuba et al (1993) that the crucial role here is played by the virtual levels which exist for positrons and heavier noble-gas atoms. So, it is the extremely large scattering length of $\mathrm{Xe}(a \approx 100 \mathrm{au})$, which determines the typical scale of the dependence of $Z_{\text {eff }}$ on the momentum: $p \sim 1 / a \approx 0.01$.

The $Z_{\text {eff }}$ value is proportional to the density of the positron wavefunction near the atom. For small momenta the $\mathrm{s}$ wavefunction of the positron just outside the atomic region can be written as $\psi=1+(f / r)$, where $f$ is the scattering amplitude and $p r \ll 1$. If a virtual level exists for the positron the scattering amplitude can be approximated using the formula for resonant scattering, $f=-1 /(\kappa+\mathrm{i} p)$ (Landau and Lifshits 1965), where $\kappa=1 / a$ is the reciprocal of the scattering length. In the region of $r \lesssim r_{\mathrm{a}}$ where the annihilation takes place the magnitude of the many-particle wavefunction is proportional to the $\psi$ above, as the two solutions are smoothly joined at the atomic boundary. If the scattering length is

$\dagger$ At this point we assume that in the annihilation rate measurements positrons are thermalized. Interestingly, it is also in the Xe gas where such thermalization is more difficult to achieve experimentally (Wright et al 1985). 
large, $a \gg r_{\mathrm{a}}$, the $f / r$ term dominates the positron wavefunction and the $p$ dependence of $Z_{\text {eff }}(10)$ can be approximated by the formula

$$
Z_{\text {eff }}(p)=\frac{K}{\kappa^{2}+p^{2}}
$$

where $K$ is a coefficient (Dzuba et al 1993). In principle, one can consider (20) as a formula for fitting $Z_{\mathrm{eff}}(p)$, with $K$ and $\kappa$ as the fit parameters. Physically, equation (20) shows that if thermal $p$ are smaller than the virtual state parameter $\kappa$ the annihilation rate is proportional to the scattering length squared. This factor explains the rapid growth of the $Z_{\text {eff }}$ values for the heavier noble gases, as we move from the Hartree-Fock values to those obtained with the full positron-atom correlation potential (rows a-c in table 2). Equation (20) fits the data very well, however, the quality of the fit was not very sensitive to changes in $\kappa$. The value of $\kappa$ determines the shape of the $Z_{\text {eff }}(p)$ curve at very small momenta, where the accuracy of our solution of the Dyson equation may be insufficient. The values found for Xe are: $K=0.288$ and $\kappa \approx-0.005$ (negative $\kappa$ corresponds to a virtual level).

Once the calculated $Z_{\text {eff }}$ values have been fit by equation (20), we can obtain a thermally averaged value of $Z_{\text {eff }}, \bar{Z}_{\text {eff }}$,

$$
\begin{aligned}
\bar{Z}_{\text {eff }} & =\int_{0}^{\infty} Z_{\text {eff }}(p) \frac{\exp \left(-p^{2} / 2 k T\right)}{(2 \pi k T)^{3 / 2}} 4 \pi p^{2} \mathrm{~d} p \\
& =\frac{K}{k T}\left\{1-\sqrt{\frac{\pi \kappa^{2}}{2 k T}} \exp \left(\kappa^{2} / 2 k T\right)\left[1-\Phi\left(\sqrt{\frac{\kappa^{2}}{2 k T}}\right)\right]\right\} \\
& \approx \frac{K}{k T} \quad \kappa \ll k T
\end{aligned}
$$

where $\Phi(x)=\frac{2}{\sqrt{\pi}} \int_{0}^{x} \mathrm{e}^{-t^{2}} \mathrm{~d} t$ is the standard error function and $k$ is Boltzman's constant.

The values of $K$ and $\kappa$ given above produce the thermally averaged value $\bar{Z}_{\text {eff }}$ for Xe that is shown in table 2 . As was discussed above, the $\kappa$ value is not very well established. However, even if it is zero, the maximum value that our calculation can produce at room temperature is $\bar{Z}_{\text {eff }}=300$.

The two formulae, (20) and (21), should enable one to learn about positron-atom (or positron-molecule) virtual or loosely bound states by studying the temperature dependence of the annihilation rate at low gas densities. Generally, if such dependence is strong at room temperature, the corresponding species must have $|\kappa|<0.045$, i.e. a binding or a virtual level energy of $25 \mathrm{meV}$ or less.

The experimental determination of the $Z_{\text {eff }}$ value of Xe is difficult for two reasons. Firstly, our calculations reveal that the scattering length of Xe for positron scattering is about -100 au. If we assume that to observe positron collisions with single atoms, each atom must occupy a cube of length 100 au, one would infer from this that it is impossible to obtain experimentally, single-atom behaviour at densities above 0.25 Amagat. It has also been shown that the presence of positrons promotes the formation of clusters (Dzuba et al 1993) by stabilizing their bound states. Therefore, unless the density is low there will not only be Xe clusters, but bound positron-atom clusters $\left(\mathrm{Xe}_{2} \mathrm{e}^{+}, \mathrm{Xe}_{3} \mathrm{e}^{+}\right.$, etc). Therefore, at densities above 0.25 Amagat, one would expect to see evidence of clustering. In annihilation experiments this would manifest as a peak in the $Z_{\text {eff }}$ versus density behaviour. Another problem in experimentally determining the $Z_{\text {eff }}$ value for Xe also related to its large scattering length (small $\kappa$ ), is the strong momentum dependence of $Z_{\text {eff. }}$. As the positron momentum goes to zero, the $Z_{\text {eff }}$ value gets very large. Since Xe is a mono-atomic species the thermalization of the positrons is slow. Any positron that is slowed down by 
the elastic collisions will quickly annihilate, thus inhibiting the complete thermalization of the positrons. Due to the strongly varying behaviour of $Z_{\text {eff }}$ with positron momentum, any deviation of the positron energy distribution from the equilibrium Maxwellian one will produce a marked difference between the 'true' $Z_{\text {eff }}$ and the experimental value.

Griffith and Heyland (1978) found that at $T=300 \mathrm{~K}$ the $Z_{\text {eff }}$ value for pure Xe at gas densities of 1-6 Amagat is $320 \pm 10$. Wright et al (1985) increased the rate of thermalization, by adding small concentrations of diatomic gases. Their results clearly show the peak in the $Z_{\text {eff }}$ versus density curve that is indicative of clustering. However, the data are very difficult to extrapolate to zero density due to the large and ill-defined slope of the points in their $Z_{\text {eff }}$ versus density graph. These problems aside, if one was to attempt to extrapolate to zero density, the zero-density value of the $Z_{\text {eff }}$ value of $\mathrm{Xe}$ is $240 \pm 60$. This value appears to be in agreement with our many-body calculations (unlike those for the other atoms where we obtained smaller $Z_{\text {eff }}$ ). There have been other calculations (McEachran et al 1980, Schrader and Svetic 1982) that suggest that the zero-density $Z_{\text {eff }}$ value of $\mathrm{Xe}$ is about 200.

Murphy and Surko (1990) measured the $Z_{\text {eff }}$ value of Xe in a modified Penning trap. The value of the density in this experiment is nine orders of magnitude smaller than in the above experiments and at these densities clustering does not occur (the measured annihilation rate $\lambda$ was indeed linear with respect to Xe density). They are able to store the positrons for up to a minute in a dilute $\mathrm{N}_{2}$ gas and there is no doubt that the positrons are completely thermalized by the time the annihilation rate is measured. They found a $Z_{\text {eff }}$ value for $\mathrm{Xe}$ of $401 \pm 20$. This result is incompatible with the previous experiments, if we accept that the peak value of $Z_{\text {eff }}=400-450$ at about 6 Amagat (Wright et al 1985) is a manifestation of clustering. A possible reason for this discrepancy could be that the positron momentum distribution may not be exactly Maxwellian in the trap. Due to the sensitivity of the $Z_{\text {eff }}$ value of $\mathrm{Xe}$ to the positron momentum, any deviation from the Maxwellian distribution would cause a different value of the measured $Z_{\text {eff }}$ value. However, in a similar positrontrap technique, Iwata et al (1995) obtained higher $Z_{\text {eff }}$ for $\mathrm{Ar}$ and $\mathrm{Kr}$ as well. For both atoms the $Z_{\text {eff }}(p)$ is relatively flat at thermal energies, so the discrepancy cannot be explained by the distorted positron momentum distribution. Thus, it appears that the true rate of positron annihilation with single $\mathrm{Xe}$ atoms is yet to be determined.

\section{Conclusions}

We have shown in this paper that the effect of virtual Ps formation is large in low-energy positron scattering from noble-gas atoms. By including virtual Ps formation as well as the polarization of the atom, we are now able to calculate accurately the total scattering cross sections. It would be very interesting to extend our scattering calculations beyond the Psformation threshold (as we have done for Mg in Gribakin and King (1996)). Experiments (see, e.g., Dababneh et al 1980) report a prominent onset in the total cross section at $E>I-6.8 \mathrm{eV}$, which, we believe, is mainly due to the ground-state Ps formation. In that case our many-body method should be able to reproduce it. It is also our next goal to perform calculations of the differential cross sections at these energies. This would enable us to make comparisons with existing experimental data (see, e.g., Kauppila et al 1994), as well as theoretical values from the optical potential calculations by Bartschat et al (1988, 1990).

The calculation of the $Z_{\text {eff }}$ values for atoms has been a more difficult problem. The inclusion of virtual Ps formation produces a large change in the calculated $Z_{\text {eff }}$ values, however, there is still a significant difference between our full many-body calculation and 
the experimental results. We now understand that the reason for the difficulty is the Coulomb enhancement of the wavefunction at small electron-positron separations. We have presented a simple method of approximating the Coulomb enhancement. This method produced good agreement with the experimental values. The method may be useful in calculating the $Z_{\text {eff }}$ values of molecules. Our approach also elucidated the role of virtual s levels which the positron forms with heavier noble-gas atoms. Information on such levels can be obtained by studying the temperature dependence of $Z_{\text {eff }}$. Despite significant efforts, there is still more experimental and theoretical work to be done before a definite $Z_{\text {eff }}$ value of $\mathrm{Xe}$ is determined. However, one must work out a proper approach to sum the electron-positron ladder-type diagram series to obtain accurate and reliable $Z_{\text {eff }}$ values.

We cannot help mentioning that there are even more surprising and puzzling results in the positron-molecule annihilation rates (see Iwata et al 1995 and references therein). Extremely large annihilation rates have been obtained for a number of large organic molecules and no explanations of these values have been suggested so far. The elegant possibility of modifying organic molecules by fluorination and watching the change of their $Z_{\text {eff }}$ can probably help to understand the mechanisms of positron-molecule annihilation or at least single out the effect of the virtual- or bound-level position on $Z_{\text {eff }}$. For example, it would seem reasonable to assume that fluorine atoms are similar to $\mathrm{Ne}$ and they attract positrons weakly (the positron scattering length for $\mathrm{Ne}$ is smaller than that for $\mathrm{H}$ ). Then, if a particular hydrocarbon molecule possesses a bound state, $\kappa>0$, the binding energy will become smaller upon fluorination of the species and the bound state would then turn into a virtual level, $\kappa<0$. This would result in a peaking behaviour of $Z_{\mathrm{eff}}$ as a function of the number of fluorines observed in experiments (Iwata and Surko 1996).

It is easy to prove theoretically that any sufficiently large molecule must form bound states with positrons (similarly to the emergence of a bound state in a system of zero-radius potential wells each of which cannot bind the positron, see e.g. Baz' et al (1971)). This is the most probable explanation of the high annihilation rate and what should be considered is the mechanism of capture. Possibly, the positron energy is transferred to vibrational or electronic degrees of freedom and very long lived resonances are formed. This is reminiscent of resonances in compound nuclei excited in neutron scattering. The energy of the neutron is distributed among different degrees of freedom and the probability is low that it will be returned to the captured neutron (in our case, positron) to give it a chance to escape. Thus, the high annihilation rates can be due to the existence of positron-molecule bound states and dense spectra of vibrational 'compound resonances' where the positron is captured.

\section{Acknowledgments}

The authors would like to thank M Yu Kuchiev and O P Sushkov for useful discussions. The support of this work by the Australian Research Council is gratefully acknowledged. This work was partially supported by the National Science Foundation through a grant for the Institute for Theoretical Atomic and Molecular Physics at Harvard University and Smithsonian Astrophysical Observatory.

\section{Appendix A. Theory of rearrangement collisions}

The purpose of this appendix is to show that the Ps-formation amplitude $\left\langle\tilde{\Psi}_{1 \mathrm{~s}, K}|V| n i\right\rangle$, equation (7), which determines the magnitude of the Ps-formation contribution to the correlation potential (4), can be derived using the rearrangement collisions theory. 
Massey and Mohr (1954) give the matrix element for Ps formation in positron-hydrogen collisions. Let us briefly re-write their derivation for the case when the wavefunction of the incoming positron is calculated in the static potential of the ground-state hydrogen atom. The total interaction in the system is

$$
W=-\frac{1}{r_{1}}+\frac{1}{r}-\frac{1}{\left|\boldsymbol{r}-\boldsymbol{r}_{1}\right|}
$$

where $r$ and $\boldsymbol{r}_{1}$ are the coordinates of the positron and electron, respectively. The interaction taken into account in the initial state of the process $\Psi_{\text {in }}=\psi_{1 \mathrm{~s}}\left(\boldsymbol{r}_{1}\right) \psi(\boldsymbol{r})$ is

$$
W_{\text {in }}=-\frac{1}{r_{1}}+\frac{1}{r}-\left\langle 1 \mathrm{~s}\left|\frac{1}{\left|\boldsymbol{r}-\boldsymbol{r}^{\prime}\right|}\right| 1 \mathrm{~s}\right\rangle
$$

where integration over $\boldsymbol{r}^{\prime}$ is assumed in the last term, which gives the electrostatic potential of the 1s electron $V_{1 \mathrm{~s}}(r)$ as experienced by the positron. The transition to the final state $\Psi_{\text {out }} \equiv \Psi_{1 \mathrm{~s}, \boldsymbol{K}}=\varphi_{1 \mathrm{~s}}\left(\boldsymbol{r}-\boldsymbol{r}_{1}\right) \exp \left[\mathrm{i} \boldsymbol{K} \cdot\left(\boldsymbol{r}+\boldsymbol{r}_{1}\right) / 2\right]$ is caused by the difference $W-W_{\text {in }}$ and in the lowest order the corresponding amplitude is given by

$$
\begin{aligned}
\left\langle\Psi_{\text {out }}\left|W-W_{\text {in }}\right| \Psi_{\text {in }}\right\rangle & =\int \Psi_{1 \mathrm{~s}, \boldsymbol{K}}^{*}\left(\boldsymbol{r}, \boldsymbol{r}_{1}\right)\left[-\frac{1}{\left|\boldsymbol{r}-\boldsymbol{r}_{1}\right|}-V_{1 \mathrm{~s}}(r)\right] \psi_{1 \mathrm{~s}}\left(\boldsymbol{r}_{1}\right) \psi(\boldsymbol{r}) \mathrm{d} \boldsymbol{r} \mathrm{d} \boldsymbol{r}_{1} \\
& =\int \tilde{\Psi}_{1 \mathrm{~s}, \boldsymbol{K}}^{*}\left(\boldsymbol{r}, \boldsymbol{r}_{1}\right)\left(-\frac{1}{\left|\boldsymbol{r}-\boldsymbol{r}_{1}\right|}\right) \psi_{1 \mathrm{~s}}\left(\boldsymbol{r}_{1}\right) \psi(\boldsymbol{r}) \mathrm{d} \boldsymbol{r} \mathrm{d} \boldsymbol{r}_{1}
\end{aligned}
$$

where

$$
\tilde{\Psi}_{1 \mathrm{~s}, \boldsymbol{K}}\left(\boldsymbol{r}, \boldsymbol{r}_{1}\right)=\Psi_{1 \mathrm{~s}, \boldsymbol{K}}\left(\boldsymbol{r}, \boldsymbol{r}_{1}\right)-\psi_{1 \mathrm{~s}}\left(\boldsymbol{r}_{1}\right) \int \psi_{1 \mathrm{~s}}^{*}\left(\boldsymbol{r}^{\prime}\right) \Psi_{1 \mathrm{~s}, \boldsymbol{K}}\left(\boldsymbol{r}, \boldsymbol{r}^{\prime}\right) \mathrm{d} \boldsymbol{r}^{\prime}
$$

is the final-state wavefunction orthogonalized to the 1 s hydrogen state, $\left|\tilde{\Psi}_{1 \mathrm{~s}, \boldsymbol{K}}\right\rangle=$ $(1-|1 \mathrm{~s}\rangle\langle 1 \mathrm{~s}|)\left|\Psi_{1 \mathrm{~s}, K}\right\rangle$. Therefore, by starting from the rearrangement collisions theory we have arrived at the Ps-formation amplitude (7), written on the many-body theory grounds.

Suppose we now consider positron impact with an $N$-electron atom. The total interaction is now

$$
W=-\sum_{i=1}^{N} \frac{Z}{r_{i}}+\frac{Z}{r}+\sum_{i<j}^{N} \frac{1}{\left|\boldsymbol{r}_{i}-\boldsymbol{r}_{j}\right|}-\sum_{i=1}^{N} \frac{1}{\left|\boldsymbol{r}-\boldsymbol{r}_{i}\right|} .
$$

In many-body theory the Hartree-Fock approximation is used to describe the ground state of the atom. The wavefunction of the initial state

$$
\Psi_{\text {in }}=\Psi_{N}\left(\boldsymbol{r}_{1}, \boldsymbol{r}_{2}, \ldots, \boldsymbol{r}_{N}\right) \psi(\boldsymbol{r})
$$

is the product of the Slater determinant $\Psi_{N}$ of the $N$ atomic electrons and the positron wavefunction calculated in the static field of the Hartree-Fock atomic ground state. The above wavefunction corresponds to the initial-state interaction

$$
W_{\text {in }}=-\sum_{i=1}^{N} \frac{Z}{r_{i}}+\frac{Z}{r}+\sum_{i=1}^{N}\left[V_{\mathrm{dir}}\left(\boldsymbol{r}_{i}\right)-V_{\mathrm{exch}}\left(\boldsymbol{r}_{i}\right)\right]-V_{\mathrm{dir}}(\boldsymbol{r})
$$

where $V_{\text {dir }}$ and $\hat{V}_{\text {exch }}$ are the direct and exchange potentials of the ground-state atom, $V_{\mathrm{dir}}\left(\boldsymbol{r}_{i}\right)=\sum_{v=1}^{N}\left\langle\nu|1 /| \boldsymbol{r}_{i}-\boldsymbol{r}^{\prime}|| \nu\right\rangle, V_{\mathrm{exch}}\left(\boldsymbol{r}_{i}\right)|\mu\rangle=\sum_{v=1}^{N} \psi_{v}\left(\boldsymbol{r}_{i}\right)\left\langle v|1 /| \boldsymbol{r}_{i}-\boldsymbol{r}^{\prime}|| \mu\right\rangle$ and $v$ runs over all electron orbitals occupied in the atomic ground state. The final state for Ps formation is now

$$
\Psi_{\text {out }}^{n}=\Psi_{N-1}^{n}\left(\boldsymbol{r}_{1}, \boldsymbol{r}_{2}, \ldots, \boldsymbol{r}_{N-1}\right) \Psi_{1 \mathrm{~s}, \boldsymbol{K}}\left(\boldsymbol{r}, \boldsymbol{r}_{N}\right)
$$


where $\Psi_{N-1}^{n}$ is the Slater determinant of the final ionic state obtained from $\Psi_{N}$ by removing an electron from a certain orbital $n$. Note that one does not have to antisymmetrize the $\Psi_{\text {out }}$ state with respect to swapping of $\boldsymbol{r}_{N}$ and $\boldsymbol{r}_{k}(k<N)$, as long as $\Psi_{\text {in }}$ is asymmetric with respect to all electron coordinates and both $W$ and $W_{\text {in }}$ are symmetric. Using equations (A6)(A9) and integrating over the $N-1$ electron coordinates one will arrive at the following answer for the Ps-formation amplitude:

$\left\langle\Psi_{\text {out }}^{n}\left|W-W_{\text {in }}\right| \Psi_{\text {in }}\right\rangle=\int \tilde{\Psi}_{1 \mathrm{~s}, \boldsymbol{K}}^{*}\left(\boldsymbol{r}, \boldsymbol{r}_{N}\right)\left(-\frac{1}{\left|\boldsymbol{r}-\boldsymbol{r}_{N}\right|}\right) \psi_{n}\left(\boldsymbol{r}_{N}\right) \psi(\boldsymbol{r}) \mathrm{d} \boldsymbol{r} \mathrm{d} \boldsymbol{r}_{N}$

where

$\tilde{\Psi}_{1 \mathrm{~s}, \boldsymbol{K}}\left(\boldsymbol{r}, \boldsymbol{r}_{N}\right)=\Psi_{1 \mathrm{~s}, \boldsymbol{K}}\left(\boldsymbol{r}, \boldsymbol{r}_{N}\right)-\sum_{v} \psi_{v}\left(\boldsymbol{r}_{N}\right) \int \psi_{v}^{*}\left(\boldsymbol{r}^{\prime}\right) \Psi_{1 \mathrm{~s}, \boldsymbol{K}}\left(\boldsymbol{r}, \boldsymbol{r}^{\prime}\right) \mathrm{d} \boldsymbol{r}^{\prime}$.

The two equations above are identical to expression (7).

\section{Appendix B. Calculation of the Ps-formation contribution}

The evaluation of equation (8) is done by expanding the atomic electron and positron wavefunctions, the Ps wavefunction and the electron-positron Coulomb interaction in terms of radial functions and spherical harmonics with respect to the nucleus. The Coulomb interaction is expanded as

$$
\frac{1}{\left|\boldsymbol{r}-\boldsymbol{r}_{1}\right|}=4 \pi \sum_{l=0}^{\infty} \frac{r_{<}^{l}}{r_{>}^{l+1}} \frac{1}{2 l+1} \sum_{m=-l}^{l} Y_{l m}^{*}(\Omega) Y_{l m}\left(\Omega_{1}\right)
$$

the Ps centre-of-mass wavefunction may be expanded using the formula

$$
\mathrm{e}^{\mathrm{i} K \cdot r / 2}=4 \pi \sqrt{\frac{\pi}{K r}} \sum_{l=0}^{\infty} \mathrm{i}^{l} J_{l+\frac{1}{2}}(K r / 2) Y_{l m}^{*}\left(\Omega_{K}\right) Y_{l m}(\Omega)
$$

and it is also useful to expand the product of the hydrogen-like wavefunction of the Ps atom and the Coulomb interaction as

$\frac{\mathrm{e}^{-\gamma\left|\boldsymbol{r}-\boldsymbol{r}_{1}\right|}}{\left|\boldsymbol{r}-\boldsymbol{r}_{1}\right|}=\frac{4 \pi}{\sqrt{r r_{1}}} \sum_{l=0}^{\infty} I_{l+\frac{1}{2}}\left(\gamma r_{<}\right) K_{l+\frac{1}{2}}\left(\gamma r_{>}\right) \sum_{m=-l}^{l} Y_{l m}^{*}(\Omega) Y_{l m}\left(\Omega_{1}\right)$

where $\gamma=\frac{1}{2}$. The last expansion needed is the one for the 1 s Ps wavefunction itself. It can be obtained from equation (B3) by calculating the derivative over $\gamma$ and using the recursion relationships of Bessel functions:

$$
\mathrm{e}^{-\gamma\left|r-r_{1}\right|}=\frac{4 \pi}{\sqrt{r r_{1}}} \sum_{l=0}^{\infty} \Phi_{l}\left(r, r_{1}\right) \sum_{m=-l}^{l} Y_{l m}^{*}(\Omega) Y_{l m}\left(\Omega_{1}\right)
$$

where

$$
\begin{aligned}
\Phi_{l}\left(r, r_{1}\right)=r_{>} & I_{l+\frac{1}{2}}\left(\gamma r_{<}\right)\left[K_{l-\frac{1}{2}}\left(\gamma r_{>}\right)+\frac{(2 l+1)}{\gamma r_{>}} K_{l+\frac{1}{2}}\left(\gamma r_{>}\right)\right] \\
& -r_{<} K_{l+\frac{1}{2}}\left(\gamma r_{>}\right)\left[I_{l-\frac{1}{2}}\left(\gamma r_{<}\right)-\frac{(2 l+1)}{\gamma r_{<}} I_{l+\frac{1}{2}}\left(\gamma r_{<}\right)\right] .
\end{aligned}
$$

After this is done the angular integrals can be evaluated analytically. Equation (B6) below then must be evaluated numerically. This is a straightforward procedure except that the convergence of the sums over angular momentum is slow. Bray and Stelbovics (1993) encountered a similar problem in their convergent close-coupling calculation of low-energy 
positron-atomic hydrogen scattering. In our calculations we included the values up to $l=7$ in the expansions. The integration over the Ps centre-of-mass momentum $K$ employed a grid of about 50 points with $\delta K=0.1$. It was sufficient to include only the outer subshell in the sum over the hole orbitals $n l$, whereas we had to include two or three outer subshells in the orthogonalization sums over $v l_{v}, v^{\prime} l_{v}^{\prime}$.

The final expression used to obtain the radial Ps-formation potential for the positron with the angular momentum $l_{i}$ is

$$
\begin{aligned}
& \Sigma_{E}^{\mathrm{Ps}}\left(r, r^{\prime}\right)=2 \pi \sum_{n} \int_{0}^{\infty} \frac{\mathrm{d} K}{E+\varepsilon_{n}-E_{1 \mathrm{~s}}-K^{2} / 4+\mathrm{i} \delta} \sum_{a c \alpha a^{\prime} c^{\prime} \alpha^{\prime}}(-1)^{\left(\alpha+\alpha^{\prime}-c-c^{\prime}\right) / 2}(2 l+1) \\
& \times(2 c+1)(2 a+1)(2 \alpha+1)\left(2 c^{\prime}+1\right)\left(2 a^{\prime}+1\right)\left(2 \alpha^{\prime}+1\right) \\
& \times \sum_{L}(2 L+1)\left(\begin{array}{ccc}
c & \alpha & L \\
0 & 0 & 0
\end{array}\right)\left(\begin{array}{ccc}
L & \alpha^{\prime} & c^{\prime} \\
0 & 0 & 0
\end{array}\right) \\
& \times\left\{\left(\begin{array}{lll}
l_{i} & a & c \\
0 & 0 & 0
\end{array}\right)\left(\begin{array}{lll}
l & a & \alpha \\
0 & 0 & 0
\end{array}\right)\left(\begin{array}{ccc}
l_{i} & a^{\prime} & c^{\prime} \\
0 & 0 & 0
\end{array}\right)\left(\begin{array}{lll}
l & a^{\prime} & \alpha^{\prime} \\
0 & 0 & 0
\end{array}\right)\left\{\begin{array}{lll}
l_{i} & L & l \\
\alpha & a & c
\end{array}\right\}\left\{\begin{array}{lll}
l_{i} & L & l \\
\alpha^{\prime} & a^{\prime} & c^{\prime}
\end{array}\right\}\right. \\
& \times R_{n}(a, c, \alpha, K, r) R_{n}\left(a^{\prime}, c^{\prime}, \alpha^{\prime}, K, r^{\prime}\right)
\end{aligned}
$$

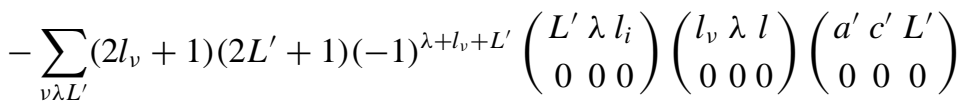

$$
\begin{aligned}
& \times\left(\begin{array}{ccc}
l_{v} & a & \alpha \\
0 & 0 & 0
\end{array}\right)\left(\begin{array}{lll}
l_{i} & a & c \\
0 & 0 & 0
\end{array}\right)\left(\begin{array}{lll}
l & a & \alpha \\
0 & 0 & 0
\end{array}\right)\left\{\begin{array}{lll}
l_{i} & L & l \\
\alpha & a & c
\end{array}\right\}\left\{\begin{array}{ccc}
l_{i} & L & l \\
\alpha^{\prime} & a^{\prime} & c^{\prime}
\end{array}\right\}\left\{\begin{array}{lll}
L^{\prime} & L & l_{v} \\
\alpha^{\prime} & a^{\prime} & c^{\prime}
\end{array}\right\} \\
& \times\left[R_{n}(a, c, \alpha, K, r) Q_{n v}\left(a^{\prime}, c^{\prime}, \alpha^{\prime}, K, r^{\prime}, \lambda\right)\right. \\
& \left.+R_{n}\left(a, c, \alpha, K, r^{\prime}\right) Q_{n v}\left(a^{\prime}, c^{\prime}, \alpha^{\prime}, K, r, \lambda\right)\right] \\
& +\sum_{v \lambda L^{\prime} \nu^{\prime} \lambda^{\prime} L^{\prime}}\left(2 l_{v}+1\right)\left(2 L^{\prime}+1\right)\left(2 l_{v}^{\prime}+1\right)\left(2 L^{\prime}+1\right)(-1)^{\lambda+\lambda^{\prime}+l_{v}+l_{v}^{\prime}+L^{\prime}+L^{\prime}} \\
& \times\left(\begin{array}{ccc}
l_{i} & \lambda & L^{\prime} \\
0 & 0 & 0
\end{array}\right)\left(\begin{array}{ccc}
L^{\prime} & a & c \\
0 & 0 & 0
\end{array}\right)\left(\begin{array}{lll}
l & \lambda & l_{v} \\
0 & 0 & 0
\end{array}\right)\left(\begin{array}{ccc}
l_{v} & a & \alpha \\
0 & 0 & 0
\end{array}\right)\left(\begin{array}{ccc}
l_{i} & \lambda^{\prime} & L^{\prime} \\
0 & 0 & 0
\end{array}\right)\left(\begin{array}{ccc}
L^{\prime} & a^{\prime} & c^{\prime} \\
0 & 0 & 0
\end{array}\right) \\
& \times\left(\begin{array}{lll}
l & \lambda^{\prime} & l_{v}^{\prime} \\
0 & 0 & 0
\end{array}\right)\left(\begin{array}{ccc}
l_{v}^{\prime} & a^{\prime} & \alpha^{\prime} \\
0 & 0 & 0
\end{array}\right)\left\{\begin{array}{ccc}
L^{\prime} & L & l_{v} \\
\alpha & a & c
\end{array}\right\}\left\{\begin{array}{ccc}
l_{i} & L & l \\
l_{v} & \lambda & L^{\prime}
\end{array}\right\}\left\{\begin{array}{lll}
L^{\prime} & L & l_{v}^{\prime} \\
\alpha^{\prime} & a^{\prime} & c^{\prime}
\end{array}\right\}\left\{\begin{array}{lll}
l_{i} & L & l \\
l_{v}^{\prime} & \lambda^{\prime} & L^{\prime}
\end{array}\right\} \\
& \left.\times Q_{n v}(a, c, \alpha, K, r, \lambda) Q_{n v^{\prime}}\left(a^{\prime}, c^{\prime}, \alpha^{\prime}, K, r, \lambda\right)\right\}
\end{aligned}
$$

where

$$
\begin{aligned}
& R_{n}(a, c, \alpha, K, r)=J_{c+\frac{1}{2}}\left(\frac{K r}{2}\right) \int_{0}^{\infty} P_{n l}\left(r_{1}\right) I_{a+\frac{1}{2}}\left(\frac{r_{>}}{2}\right) K_{a+\frac{1}{2}}\left(\frac{r_{<}}{2}\right) J_{\alpha+\frac{1}{2}}\left(\frac{K r_{1}}{2}\right) \mathrm{d} r_{1} \\
& Q_{n v}(a, c, \alpha, K, r, \lambda)=J_{c+\frac{1}{2}}\left(\frac{K r}{2}\right) V_{n v}^{(\lambda)}(r) \int_{0}^{\infty} P_{v l_{v}}\left(r_{1}\right) \Phi_{a}\left(r, r_{1}\right) J_{\alpha+\frac{1}{2}}\left(\frac{K r_{1}}{2}\right) \mathrm{d} r_{1}
\end{aligned}
$$

and

$$
V_{n v}^{(\lambda)}(r)=\int_{0}^{\infty} P_{\nu l_{v}}\left(r_{1}\right) \frac{r_{<}^{\lambda}}{r_{>}^{\lambda+1}} P_{n l}\left(r_{1}\right) \mathrm{d} r_{1} .
$$

\section{References}

Akhiezer A I and Berestetskii V B 1965 Quantum Electrodynamics (New York: Interscience) Amusia M Ya and Cherepkov N A 1975 Case Stud. At. Phys. 5 47-179

Amusia M Ya, Cherepkov N A, Chernysheva L V, Davidovic D M and Radojevic V 1982 Phys. Rev. A 25219 
Amusia M Ya, Cherepkov L V, Chernysheva L V and Shapiro S G 1976 J. Phys. B: At. Mol. Phys. 9 L531-4

Bartschat K, McEachran R P and Stauffer A D 1988 J. Phys. B: At. Mol. Opt. Phys. 21 2789-800

-1990 J. Phys. B: At. Mol. Opt. Phys. 23 2349-55

Basu M, Mukherjee M and Ghosh A S 1989 J. Phys. B: At. Mol. Opt. Phys. 23 2195-204 1990 J. Phys. B: At. Mol. Opt. Phys. 23 2641-8

Baz' A I, Zeldovich Ya B and Perelomov A M 1971 Scattering, Reactions and Decays in Nonrelativistic Quantum Mechanics (Moscow: Nauka) p 30

Bell J S and Squires E J 1959 Phys. Rev. Lett. 396

Bhatia A K, Temkin A and Eiserike H 1974 Phys. Rev. A 9223

Bray I and Stelbovics A T 1993 Phys. Rev. A 48 4787-9

Canter K F and Roellig L O 1975 Phys. Rev. A 12 386-95

Chernysheva L V, Gribakin G F, Ivanov V K and Kuchiev M Yu 1988 J. Phys. B: At. Mol. Opt. Phys 21 L419-25

Coleman P G Griffith T C Heyland G R and Killeen T L 1975 J. Phys. B: At. Mol. Phys. 8 1734-43

Dababneh M S, Kauppila W E, Downing J P, Laperriere F, Pol V, Smart J H and Stein T S 1980 Phys. Rev. A 22 1872-7

Dzuba V A, Flambaum V V, Gribakin G F and King W A 1995 Phys. Rev. A 52 4541-6

Dzuba V A, Flambaum V V, Gribakin G F and Sushkov O P 1991 Phys. Rev. A 442823.

Dzuba V A, Flambaum V V, King W A, Miller B N and Sushkov O P 1993 Phys. Scr. T 46 248-51

Dzuba V A, Flambaum V V, Silvestrov P G and Sushkov O P 1987 J. Phys. B: At. Mol. Phys. 201399

Dzuba V A, Flambaum V V and Sushkov O P 1989 Phys. Lett. 140A 493

Dzuba V A and Gribakin G F 1994a Phys. Rev. A $492483-92$

-1994b Phys. Rev. A 50 3551-2

Gribakin G F, Gultsev B V, Ivanov V K, Kuchiev M Yu 1990 J. Phys. B: At. Mol. Opt. Phys. 23 4505-19

Gribakin G F and King W A 1994 J. Phys. B: At. Mol. Opt. Phys. 27 2639-45

1996 Can. J. Phys. 74 at press

Griffith T C and Heyland G R 1978 Phys. Rep. 39 169-277

Hewitt R N, Noble C J and Bransden B H 1990 J. Phys. B: At. Mol. Opt. Phys. 23 4185-95

-1993 J. Phys. B: At. Mol. Opt. Phys. 26 3661-77

Higgins K and Burke P G 1993 J. Phys. B: At. Mol. Opt. Phys. 26 4269-88

Humberston J W 1973 J. Phys. B: At. Mol. Phys. 6 L305-8

-1974 J. Phys. B: At. Mol. Phys. 7 L286-9

Iwata K, Greaves R G, Murphy T J, Tincle M D and Surko C M 1995 Phys. Rev. A 51 473-87

Iwata K and Surko C M 1996 Private communication

Jiang J, Surdutovich A, Kauppila W E, Kwan C K, Stein T S and Zhou S 1996 Can. J. Phys. at press

Kauppila W E, Dou L, Kwan C K, Przybyla D and Stein T S 1994 Hyperfine Interactions 89 143-60

Kelly H P 1963 Phys. Rev. 131 684-99

Kernoghan A A, McAlinden M T and Walters H R J 1994 J. Phys. B: At. Mol. Opt. Phys. 27 L211

Kuchiev M Yu 1992 Private communication

Landau L D and Lifshitz E M 1965 Quantum Mechanics (Oxford: Pergamon)

McAlinden M T, Kernoghan A A and Walters H R J 1994 Hyperfine Interactions 89 161-94

-1996 J. Phys. B: At. Mol. Opt. Phys. 29 555-69

McEachran R P, Morgan D L, Ryman A G and Stauffer A D 1977 J. Phys. B: At. Mol. Phys. 10 $663-77$ 1978a J. Phys. B: At. Mol. Phys. 11 951-3

McEachran R P, Ryman A G and Stauffer A D 1978b J. Phys. B: At. Mol. Phys. 11 551-61

-1979 J. Phys. B: At. Mol. Phys. 12 1031-41

McEachran R P, Stauffer A D and Campbell L E M 1980 J. Phys. B: At. Mol. Phys. 13 1281-92

Massey H S W and Mohr C B O 1954 Proc. Phys. Soc. A 67 695-704

Migdal A B 1967 Theory of Finite Fermi-Systems and Applications to Atomic Nuclei (New York: Interscience)

Mitroy J 1993 Aust. J. Phys. 46 751-71

1994 Phys. Rev. Lett. 73 2966-9

Murphy T J and Surko C M 1990 J. Phys. B: At. Mol. Opt. Phys. 23 L727-32

Radtsig A A and Smirnov B M 1986 Handbook: Parameters of Atoms and Atomic Ions (Moscow: Energoatomizdat)

Schrader D M and Svetic R E 1982 Can. J. Phys. 60 517-42

Sinapius G, Raith W and Wilson W G 1980 J. Phys. B: At. Mol. Phys. 13 4079-90

Tuomisaari M, Rytsölä K and Hautojärvi P 1988 J. Phys. B: At. Mol. Opt. Phys. 21 3917-28

Weast R C 1988 CRC Handbook of Physics and Chemistry 69th edn (Boca Raton, FL: CRC Press)

Wright G L, Charlton M, Griffith T C and Heyland G R 1985 J. Phys. B: At. Mol. Phys. $184327-47$ 